\title{
EXAFS spectroscopy: a powerful tool for the study of local vibrational dynamics
}

\author{
Andrea Sanson \\ Department of Physics and Astronomy, University of Padova, Padova I-35131, Italy.
}

Correspondence to: Prof. Andrea Sanson, Department of Physics and Astronomy, University of Padova, Padova I-35131, Italy. Email: andrea.sanson@unipd.it

How to cite this article: Sanson A. EXAFS spectroscopy: a powerful tool for the study of local vibrational dynamics. Microstructures 2021;1:2021004. http://dx.doi.org/10.20517/microstructures.2021.03

Received: 19 Jul 2021 First Decision: 11 Aug 2021 Revised: 24 Aug 2021 Accepted: 27 Aug 2021 Published: 27 Aug 2021

Academic Editor: Jun Chen, Shujun Zhang Copy Editor: Yue-Yue Zhang Production Editor: Yue-Yue Zhang

\begin{abstract}
Extended X-ray absorption fine structure (EXAFS) spectroscopy is an ideal technique for studying the local vibrational dynamics of materials due to its sensitivity to short-range order, correlation of atomic motion and anharmonicity. However, despite this, EXAFS is widely employed to investigate the local structure but its use in the study of local dynamics is far more limited. In this brief review, the potential of EXAFS as a vibrational probe is presented with the aim of promoting its application in the study of the local dynamics of solid-state materials.
\end{abstract}

Keywords: EXAFS, local dynamics, thermal disorder

\section{INTRODUCTION}

Knowledge of vibrational dynamics is fundamental for understanding the physical properties of materials. Various experimental and theoretical approaches are used to study vibrational dynamics in materials, ranging from Bragg diffraction to vibrational spectroscopy ${ }^{[1-6]}$, or from $a b$ initio calculations to molecular dynamics $^{[7-9]}$. From an experimental perspective, X-ray and neutron diffraction can give precise parameters that describe the dynamic (but also static) displacements of atoms in crystals, such as mean-square atomic displacements ${ }^{[10,11]}$. Inelastic neutron and X-ray scattering can be used to measure phonon dispersion curves $\omega(k)$, i.e., the dependence of phonon frequency $\omega$ on its wavevector $\mathrm{k}^{[12-14]}$. Nuclear inelastic scattering, although not appropriate as with the latter two techniques in the measurement of dispersion curves, provides a direct measurement of the density of phonon states in shorter times ${ }^{[15]}$. Vibrational spectroscopy, such as in- 
frared or Raman spectroscopy, allows for the determination of the vibrational frequency of selected vibrational modes at the center of the Brillouin zone ${ }^{[5,6,16,17]}$.

Alternatively, extended X-ray absorption fine structure (EXAFS) spectroscopy ${ }^{[18]}$ provides both unique and complementary information compared to that obtained from the techniques described above. In fact, in addition to being sensitive to short-range order and atomic sites, EXAFS is particularly sensitive to the correlation of vibrational motion, both parallel and perpendicular to the bond direction, and anharmonicity ${ }^{[19-22]}$. However, although these properties make EXAFS unique, it is largely employed as a structural probe rather than as a dynamic one.

In this brief review, the capabilities of EXAFS spectroscopy for the study of local dynamics are reported. The intention is to present, for non-specialists, an introduction to the use of EXAFS in studying the vibrational dynamics of materials. For readers who wish to deepen their knowledge of EXAFS, other more exhaustive references are available ${ }^{[23-25]}$.

The review is organized as follows. Section 2 contains a synthetic introduction to the theory of EXAFS and the cumulants method. Section 3 shows a comparison between EXAFS spectroscopy and Bragg diffraction. The relations connecting EXAFS parameters to atomic thermal displacements and the sensitivity of EXAFS to atomic correlation are also presented here. Section 4 shows the phenomenological relationship of EXAFS to anharmonicity, emphasizing the differences between the one-dimensional potential probed by EXAFS and the three-dimensional crystal potential. Section 5 provides some recommendations regarding the data analysis procedure to obtain more reliable results. Section 6 provides some examples of studies that show the potential of EXAFS in the study of local dynamics. Section 7 presents the conclusions of this review.

\section{FUNDAMENTALS OF EXAFS}

The aim of an EXAFS experiment is to measure the X-ray absorption coefficient of a selected atomic species as a function of energy [Figure $1 \mathrm{~A}$ ]. After the absorption edge, the absorption coefficient $\mu(E)$ exhibits some oscillations. The oscillations extending from $\sim 30-50 \mathrm{eV}$ up to $1000 \mathrm{eV}$ and more above the edge represent the so-called EXAFS oscillations and can be explained from a phenomenological point of view, as depicted in Figure $1 \mathrm{~B}$. When an incoming X-ray photon of energy $E$ is absorbed, a core electron of binding energy $E_{0}$ is emitted (known as a photoelectron) with kinetic energy $E-E_{0}$. The ejected photoelectron can be described as a spherical wave propagating around the absorbing atom. This outgoing wave is scattered by the neighboring atoms and produces scattered waves that can interfere (constructively or destructively) with the outgoing wave. These interferences give rise to the EXAFS oscillations. As a result, EXAFS spectroscopy can give information regarding the interatomic distances between the absorbing atom and its neighbors, the type and number of neighbors, as well as their structural and thermal disorder ${ }^{[25,26]}$. The region between the absorption edge and $\sim 30-50 \mathrm{eV}$ is instead the X-ray absorption near edge structure (XANES) region, which gives information regarding the valence state and chemical coordination of the absorbing atom ${ }^{[27]}$. XANES is not considered here because it is beyond the scope of this review.

The EXAFS signal is defined as:

$$
\chi(k)=\frac{\mu-\mu_{0}}{\Delta \mu_{0}}
$$

where $\mu$ is the measured oscillating absorption coefficient, $\mu_{0}$ is the smooth absorption coefficient of the isolated atom, estimated by polynomial splines, and $\Delta \mu_{0}$ is the measured edge jump ${ }^{[24,28]}$. The EXAFS signal is conveniently expressed as a function of the photoelectron wavenumber $k=\sqrt{2 m\left(E-E_{0}\right) / \hbar^{2}}$, where, as mentioned above, $E$ is the X-ray photon energy and $E_{0}$ is the binding energy of the core electron. As an example, 


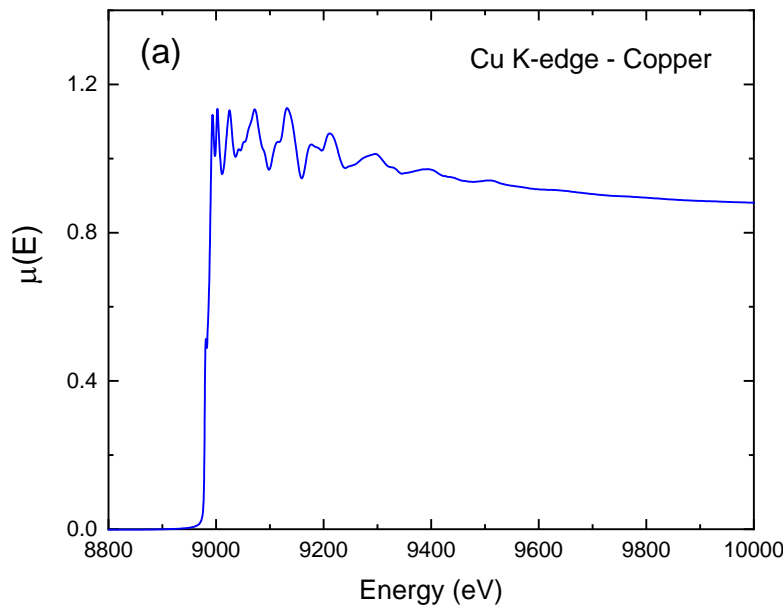

(b)

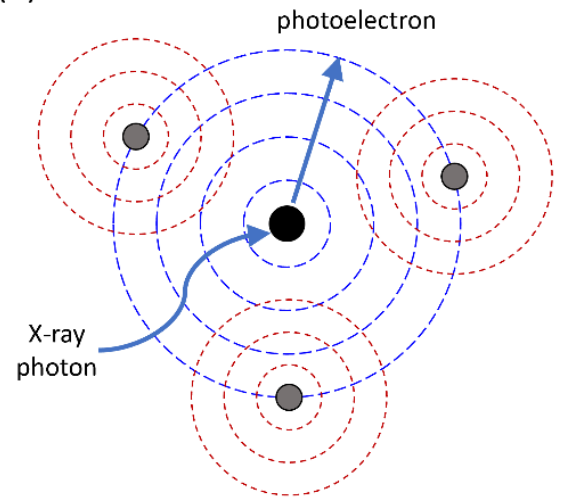

Figure 1. (A) X-ray absorption coefficient (measured at the Cu K-edge of metallic copper at room temperature) plotted as a function of the incident beam energy. (B) Schematic view of EXAFS mechanism.
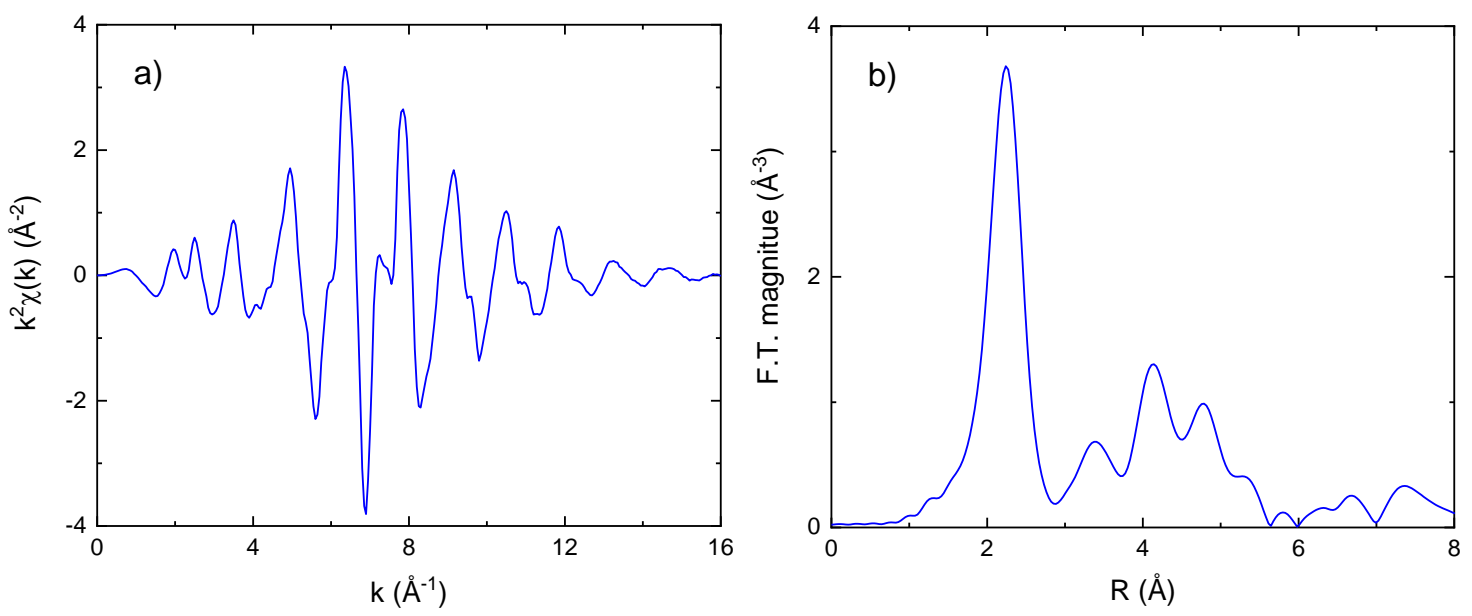

Figure 2. $\mathrm{k}^{2}$-weighted EXAFS signal extracted from the absorption coefficient $\mu(E)$ shown in Figure $1 \mathrm{~A}$ (panel $\mathrm{A}$ ) and the corresponding Fourier transform (panel B). The first F. T. peak is due to backscattering from the nearest neighboring Cu atoms.

Figure 2A shows the EXAFS signal extracted from the absorption coefficient reported in Figure 1A. The overall EXAFS signal is the result of different single and multiple scattering processes of the EXAFS photoelectron. The most dominant contribution is from the backscattering of the photoelectron by neighboring atoms, as can be observed by the Fourier transform of the EXAFS signal shown in Figure 2B.

The starting point of EXAFS theory ${ }^{[24,25,28]}$ is Fermi's golden rule within the dipole approximation, according to which the absorption coefficient can be written as:

$$
\mu \propto \rho\left(E_{F}\right)\left|\left\langle\psi_{i}|\bar{\varepsilon} \cdot \hat{r}| \psi_{f}\right\rangle\right|^{2}
$$

where $\rho\left(E_{F}\right)$ is the density of the final electronic states, which is constant in the EXAFS region, $\bar{\varepsilon} \cdot \hat{r}$ is the dot product of the electric field with the direction of the electron position vector and $\left|\psi_{i}\right\rangle$ and $\left|\psi_{f}\right\rangle$ are the initial and final electronic states, respectively. Working in the planewave and single electron approximation, from Equation (2), the EXAFS signal for the single scattering, by assuming the atoms are frozen in their equilibrium positions, takes the form:

$$
\chi(k)=\frac{S_{0}^{2}}{k R^{2}} N|f(k)| e^{-2 R / \lambda(k)} \sin [2 k R+\varphi(k)]
$$


where $R$ and $N$ are the interatomic distance and coordination number, respectively, $S_{0}{ }^{2}$ is a loss factor (typically between 0.7 and 0.9) due to intrinsic inelastic effects in the absorbing atom, $\lambda(k)$ is the photoelectron mean free path (typically 6 to $12 \AA$ ) and $f(k)$ and $\varphi(k)$ are the photoelectron scattering path amplitude and phase shift, respectively. Equation (3) does not consider atomic vibrations. The first method to include thermal (but also static) disorder is to introduce the Debye-Waller factor $e^{-2 k^{2} \sigma^{2}}$, which considers the exponential damping of the EXAFS signal related to the broadening of the atom-pair distance distribution. $\sigma$ represents the standard deviation of this distance distribution, which is assumed to be Gaussian here. This results in the so-called "standard formula of EXAFS":

$$
\chi(k)=\frac{S_{0}^{2}}{k R^{2}} N|f(k)| e^{-2 R / \lambda(k)} e^{-2 k^{2} \sigma^{2}} \sin [2 k R+\varphi(k)]
$$

However, thermal and structural disorder, including anharmonicity, can be better considered by removing the Gaussian approximation and by introducing a general one-dimensional distribution of distances between the absorbing and scattering atoms, $\rho(r)$. It can be demonstrated that the single-shell EXAFS signal is connected with the distance distribution $\rho(r)$ by ${ }^{[25]}$ :

$$
\chi(k)=\frac{S_{0}^{2} N}{k} \operatorname{Im}\left\{f(k) e^{i \varphi(k)} \int_{0}^{+\infty} \rho(r) \frac{e^{-2 r / \lambda(k)}}{r^{2}} e^{2 i k r} d r\right\}
$$

Note that, due to the spherical-wave nature of the EXAFS photoelectron and its limited mean free path $\lambda$, EXAFS does not probe the real distance distribution $\rho(r)$ but an effective distance distribution $P(r, \lambda)$ :

$$
P(r, \lambda)=\rho(r) \frac{e^{-2 r / \lambda}}{r^{2}}
$$

The integral in Equation (5) represents the structural part of the EXAFS formula and its logarithm can be developed in a Maclaurin series to obtain the final EXAFS formula, which is particularly suitable for the study of dynamics ${ }^{[25,29,30]}$ :

$$
\chi(k)=\frac{S_{0}^{2} N}{k}|f(k)| \frac{e^{-2 C_{1} / \lambda}}{C_{1}^{2}} e^{-2 k^{2} C_{2}+\frac{2}{3} k^{4} C_{4} \cdots} \sin \left[2 k C_{1}-\frac{4}{3} k^{3} C_{3} \ldots+\varphi(k)\right]
$$

where the parameters $C_{i}$ are the cumulants of the effective distribution $P(r, \lambda)$. The lowest-order cumulants have a direct physical meaning: $C_{1}$ and $C_{2}$ are the mean value $\langle r\rangle$ and variance $\left\langle(r-\langle r\rangle)^{2}\right\rangle$ of the distribution $P(r, \lambda)$, respectively; $C_{3}=\left\langle(r-\langle r\rangle)^{3}\right\rangle$ and measures its asymmetry; $C_{4}=\left\langle(r-\langle r\rangle)^{4}\right\rangle-3 C_{2}{ }^{2}$ and measures its flatness with respect to a Gaussian distribution. Cumulants higher than the second order are zero for Gaussian distributions. In this case, Equation (7) reduces to the standard formula of EXAFS given by Equation (4). Cumulants higher than the fourth order are generally omitted in order to not introduce too many fitting parameters.

As stated above, the EXAFS cumulants $C_{i}$ in Equation (7) refer to the effective distance distribution $P(r, \lambda)$. However, we are interested in obtaining information on the real distance distribution $\rho(r)$. The first cumulant $C_{1}{ }^{*}$ of $\rho(r)$ (i.e., its average value) can be derived from the expression:

$$
C_{1}{ }^{*} \cong C_{1}+\frac{2 C_{2}}{C_{1}}\left(1+\frac{C_{1}}{\lambda}\right)
$$

while the higher order cumulants, $C_{i}{ }^{*}$ for $i>1$, correspond to a very good approximation to the cumulants $C_{i}$ of the effective distance distribution $P(r, \lambda)$ :

$$
C_{2}{ }^{*} \cong C_{2} ; C_{3}{ }^{*} \cong C_{3} ; C_{4}{ }^{*} \cong C_{4} ; \ldots
$$




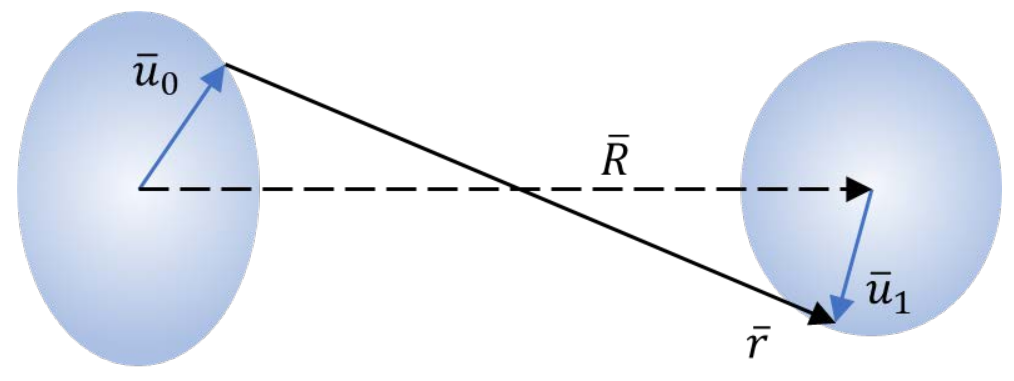

Figure 3. Instantaneous atomic displacements $\bar{u}_{0}$ and $\bar{u}_{1}$, instantaneous interatomic distance $\bar{r}$ and distance $\bar{R}$ between the equilibrium positions. EXAFS probes the instantaneous distance $r$, while Bragg diffraction measures the equilibrium distance $R$.

The exact relationships between $C_{i}{ }^{*}$ and $C_{i}$ are reported in Ref. ${ }^{[30]}$. As detailed below, the interatomic distance $r$ probed by EXAFS is different from the interatomic distance measured by Bragg diffraction. This is the fundamental reason that makes EXAFS an exceptional technique in exploring the local dynamics of condensed matter systems.

\section{EXAFS VS. DIFFRACTION FOR ASSESSING LOCAL DYNAMICS}

EXAFS spectroscopy and X-ray (or neutron) diffraction are complementary techniques.

- Owing to the limited mean free path of the EXAFS photoelectron, EXAFS is sensitive to short-range order, typically up to $\sim 10 \AA$. In contrast, diffraction is sensitive to the long-range order.

- Because the mean lifetime of the EXAFS photoelectron $\left(\sim 10^{-15}-10^{-16} \mathrm{~s}\right)$ is much shorter than the typical times of the atomic vibrations $\left(\sim 10^{-12} \mathrm{~s}\right)$, EXAFS probes the atomic distances by taking a picture of the instantaneous interatomic distances. In contrast, Bragg diffraction probes the average atomic positions. Therefore, indicating with $\bar{r}_{0}$ and $\bar{r}_{1}$ the instantaneous atomic positions of a given pair of atoms, EXAFS measures the distance $\langle r\rangle=\left\langle\left|\bar{r}_{0}-\bar{r}_{1}\right|\right\rangle$, named as the true bond distance, while diffraction measures the difference between the average atomic positions, $R=\left|\left\langle\bar{r}_{1}\right\rangle-\left\langle\bar{r}_{0}\right\rangle\right|$, named as the apparent bond distance.

- Finally, EXAFS measures the relative atomic vibrations, including the correlation of atomic motion, while diffraction only measures uncorrelated atomic vibrations from the atomic thermal factors included in the Rietveld analysis.

Let us now consider a pair of atoms, where the absorber atom of EXAFS and one of its neighbors are labeled as 0 and 1 , respectively. Let $\bar{u}_{0}$ and $\bar{u}_{1}$ be their instantaneous thermal displacements from their equilibrium positions, $\bar{r}$ the instantaneous interatomic distance and $\bar{R}$ the distance between the equilibrium positions [Figure 3]. Indicating with $\Delta \bar{u}=\bar{u}_{1}-\bar{u}_{0}$ the relative thermal displacement of the pair of considered atoms, it can be written:

$$
\bar{r}=\bar{R}+\Delta \bar{u}
$$

and considering the projections of the relative displacement $\Delta \bar{u}$ parallel and perpendicular to the bond direction $\hat{R}$ :

$$
\Delta u_{\|}=\Delta \bar{u} \cdot \hat{R} \quad\left(\Delta u_{\perp}\right)^{2}=(\Delta u)^{2}-\left(\Delta u_{\|}\right)^{2}
$$

the scalar distance $r$ can be approximated to:

$$
r \cong R+\Delta u_{\|}+\frac{\left(\Delta u_{\perp}\right)^{2}}{2 R}-\frac{\Delta u_{\|}\left(\Delta u_{\perp}\right)^{2}}{2 R^{2}}
$$


and thus its average value results in:

$$
\langle r\rangle \cong R+\left\langle\Delta u_{\|}\right\rangle+\frac{\left\langle\left(\Delta u_{\perp}\right)^{2}\right\rangle}{2 R}-\frac{\left\langle\Delta u_{\|}\left(\Delta u_{\perp}\right)^{2}\right\rangle}{2 R^{2}}
$$

For harmonic displacements $\left\langle\Delta u_{\|}\right\rangle=0$ and $\left\langle\Delta u_{\|}\left(\Delta u_{\perp}\right)^{2}\right\rangle=0$, Equation (13) reduces to ${ }^{[31]}$ :

$$
\langle r\rangle \cong R+\frac{\left\langle\left(\Delta u_{\perp}\right)^{2}\right\rangle}{2 R}
$$

The final term in Equation (14) is proportional to the atomic mean square relative displacement (MSRD) perpendicular to the bond direction, $\left\langle\Delta u_{\perp}{ }^{2}\right\rangle$, which is always positive. The average bond distance $\langle r\rangle$ measured by EXAFS $\left(C_{1}{ }^{*}\right)$ is thus always larger than the crystallographic bond distance $R$ measured by Bragg diffraction. As a consequence, EXAFS and diffraction give different and complementary information on the interatomic distances. In particular, by inverting Equation (14), one can obtain the perpendicular MSRD for a given pair of atoms ${ }^{[21,32,33]}$. Quantitative examples are provided in the next sections.

The second cumulant of EXAFS, $C_{2}{ }^{*}$, corresponds to the variance $\sigma^{2}=\left\langle(r-\langle r\rangle)^{2}\right\rangle$ of the real distribution $\rho(r)$. It is straightforward to show that, to the first order, the variance of $\rho(r)$ corresponds to the parallel $\operatorname{MSRD}^{[19,30]}$ :

$$
C_{2}{ }^{*}=\left\langle(r-\langle r\rangle)^{2}\right\rangle \cong\left\langle\left(\Delta u_{\|}\right)^{2}\right\rangle
$$

which can be developed as:

$$
\left\langle\left(\Delta u_{\|}\right)^{2}\right\rangle=\left\langle\left[\left(\bar{u}_{1}-\bar{u}_{0}\right) \cdot \hat{R}\right]^{2}\right\rangle=\left\langle\left(\bar{u}_{0} \cdot \hat{R}\right)^{2}\right\rangle+\left\langle\left(\bar{u}_{1} \cdot \hat{R}\right)^{2}\right\rangle-2\left\langle\left(\bar{u}_{0} \cdot \hat{R}\right)\left(\bar{u}_{1} \cdot \hat{R}\right)\right\rangle
$$

The first two terms on the right-hand side of Equation (16) are the atomic mean square displacements (MSDs) of the absorber and backscatterer atoms along the bond direction, respectively, which can be obtained from Bragg diffraction. The third and final term in Equation (16) is known as the displacement correlation function (DCF) and is a measure of the degree of correlation of vibrational motion along the bond direction. As a result, the stronger the in-phase vibrational motion, the smaller the parallel $\operatorname{MSRD}\left\langle\Delta u_{\|}{ }^{2}\right\rangle$. The correlation term DCF decreases with increasing distance and disappears for the long distances of the outer shells. It is clear that the comparison between the parallel MSRD $\left(C_{2}{ }^{*}\right)$, measured by EXAFS, and the MSD measured by diffraction, allows us to derive unique information on the correlation of atomic motion along the bond direction and, consequently, to evaluate the stiffness of the chemical bond ${ }^{[34,35]}$.

Finally, once the parallel and perpendicular MSRDs are known, we can determine the anisotropy of the relative thermal vibrations through the ratio:

$$
\gamma=\frac{\left\langle\Delta u_{\perp}^{2}\right\rangle}{\left\langle\Delta u_{\|}^{2}\right\rangle}
$$

which depends on the particular dynamic properties of the system. In the case of parallel-perpendicular isotropy, $\gamma=2$. For example, the experimental value of $\gamma$ found for the first shell of copper is slightly larger than 2 , indicating weak parallel-perpendicular anisotropy ${ }^{[36]}$. A higher value of $\gamma \cong 6$ was instead found for the first shell of germanium, in agreement with theoretical calculations ${ }^{[37,38]}$. The different anisotropy between germanium and copper, which can be attributed to the contribution of transverse optical modes, cannot be detected by Bragg diffraction. Indeed, owing to the crystal symmetry of copper and germanium, the atomic MSDs measured by diffraction have to be isotropic in both cases. 
In summary, EXAFS provides unique information on the local vibrational dynamics. This is because EXAFS measures the true bond distance, including the correlation of the atomic thermal vibrations, while Bragg diffraction measures the apparent bond distance, where the correlation is not included. The relationship between EXAFS and diffraction distances, given by Equation (14), allows us to determine the perpendicular MSRD of a given pair of atoms. The second cumulant of EXAFS, instead, allows us to determine the parallel MSRD by Equation (15) and, ultimately, the anisotropy of the relative thermal vibrations. The practical procedure for obtaining this information is presented in Section 5.

\section{ANHARMONICITY}

The distribution $\rho(r)$ of the interatomic distances probed by EXAFS contains information on the anharmonicity. In the classical approximation, the distribution $\rho(r)$ is connected to a one-dimensional effective pair potential $V(r)$ through the canonical average ${ }^{[39]}$ :

$$
\rho(r, T)=\frac{e^{-V(r) / k_{B} T}}{\int e^{-V(r) / k_{B} T} d r}
$$

where $k_{B}$ is the Boltzmann constant. $V(r)$ is not the real interatomic potential between the pair of atoms, but depends on the overall behavior of all the atoms in the crystal and is, in principle, temperature dependent, both in position and shape. In the hypothesis that $V(r)$ is independent of temperature, the first four cumulants of $\rho(r, T)$ can be expressed as a function of the force constants of the effective potential $V(r)$ by relations ${ }^{[40,41]}$ :

$$
\begin{aligned}
& \Delta C_{1}{ }^{*}=-\left(3 b / a^{2}\right) k_{B} T+\ldots \\
& C_{2}{ }^{*}=\left(k_{B} T / a\right)+\left(k_{B} T / a\right)^{2}\left[(6 b / a)^{2}-(12 c / a)\right]+\ldots \\
& C_{3}{ }^{*}=-\left(k_{B} T / a\right)^{2}(6 b / a)+\ldots \\
& C_{4}{ }^{*}=\left(k_{B} T / a\right)^{3}\left[\left(108 b^{2} / a^{2}\right)-(24 c / a)\right]+\ldots
\end{aligned}
$$

where, indicating with $u$ the variation of interatomic distance with respect to the potential minimum, the effective potential has been expanded as:

$$
V(u)=a u^{2} / 2+b u^{3}+c u^{4}+\ldots
$$

Note that, to the first order and in the classical approximation, the first and second cumulants grow linearly with temperature [Equations (19) and (20)], while the third and fourth cumulants grow, respectively, with the square and cube of the temperature [Equations (21) and (22)]. As an example, Figure 4 shows the first four cumulants of the first Se-Cd coordination shell of CdSe measured by EXAFS and calculated by classic molecular dynamics simulations ${ }^{[42,43]}$. Analogous results were found in other compounds, such as copper or gold [Figure 5] ${ }^{[36,44]}$. Equation (20) also shows the second-order anharmonic correction to $C_{2}{ }^{*}$, which has been observed, for example, in crystalline germanium ${ }^{[22]}$. In the case that anharmonic effects are negligible and $V(u)$ is a simple parabolic function, $b=0$ and $c=0$, and consequently the third and fourth cumulants are zero. However, it is clear that in most cases, the anharmonicity cannot be neglected and the EXAFS cumulants higher than the second-order allow us to consider these anharmonic effects that, as discussed in the next section, are important to obtain accurate results for the first and second cumulants. From Equations (19)-(21), it is trivial to prove that the bond thermal expansion, to the first order, is the result of the potential asymmetry through:

$$
\Delta C_{1}{ }^{*}=\frac{C_{3}{ }^{*}}{2 C_{2}{ }^{*}}
$$




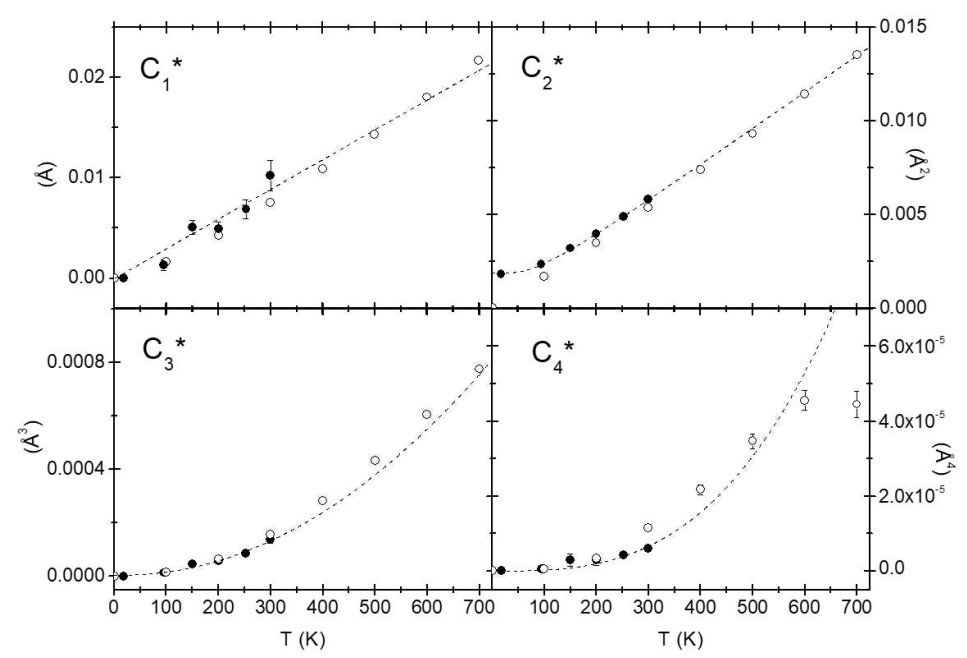

Figure 4. First four cumulants of the first Se-Cd coordination shell of CdSe measured by EXAFS (solid circles) and calculated by classic molecular dynamics simulations (open circles) [43]. The dashed lines show the linear behavior for the first and second cumulants, in the classical limit, and the quadratic and cubic behavior for the third and fourth cumulants, respectively. (c) IOP Publishing. Reproduced with permission. All rights reserved.

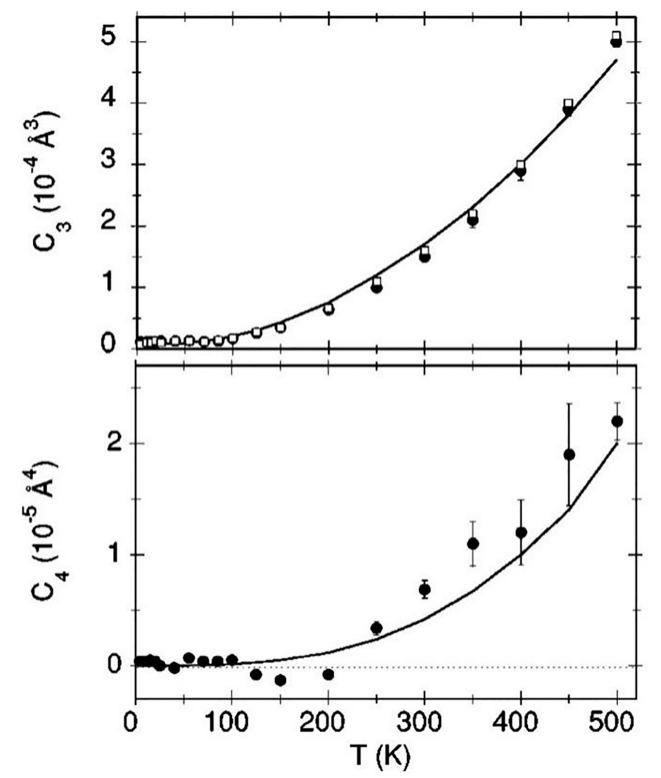

Figure 5. Third (upper panel) and fourth (lower panel) cumulants measured by EXAFS for the first Cu-Cu coordination shell of copper. The continuous lines are the best fitting theoretical model with $T^{2}$ and $T^{3}$ functions, respectively. Figure taken from Ref. [36]. (C) APS Publishing. Reproduced with permission. All rights reserved.

However, in the treatment presented above, the effective pair potential $V(r)$ was considered independent of temperature, but in reality, this is not true. In this regard, let us consider a pair of atoms in a perfect harmonic crystal at an equilibrium distance of $R$. In the case of isotropic thermal vibrations with $\sigma \ll R$, where $\sigma$ is the standard deviation of the relative thermal displacements along a direction, it can be shown that the corresponding distribution $\rho(r)$ of the interatomic distances assumes the form ${ }^{[30]}$ :

$$
\rho(r) \cong \frac{1}{\sqrt{2 \pi} \sigma} \frac{r}{R} e^{-\frac{(r-R)^{2}}{2 \sigma^{2}}}
$$


Therefore, even in a harmonic crystal, the one-dimensional real distribution $\rho(r)$ probed by EXAFS is not Gaussian. This is due to the atomic vibrations perpendicular to the bond direction. Indeed, when the perpendicular vibrations are negligible, it results that $r \cong R$ (see Figure 3) and Equation (25) reduces to a Gaussian distribution. From Equation (25), using the definition of central moments, it is possible to calculate the corresponding first three cumulants:

$$
\begin{aligned}
& C_{1}{ }^{*} \cong R+\frac{\sigma^{2}}{R} \\
& C_{2}{ }^{*} \cong \sigma^{2} \\
& C_{3}{ }^{*} \cong \frac{2 \sigma^{6}}{R^{3}}
\end{aligned}
$$

and the position of the maximum distribution thus obtaining:

$$
r_{\max }=\frac{R+\sqrt{R^{2}+4 \sigma^{2}}}{2} \cong R+\frac{\sigma^{2}}{R}
$$

Equations (26) and (27) correspond to Equations (14) and (15), respectively, because in the case of isotropy, $\left\langle\Delta u_{\perp}{ }^{2}\right\rangle / 2=\left\langle\Delta u_{\|}{ }^{2}\right\rangle=\sigma^{2}$. More importantly, by comparing Equations (26) and (29), the average position $C_{1}{ }^{*}$ corresponds to the position of the maximum distribution, $r_{\max }$, and both grow with temperature, since in the classical limit, $\sigma^{2} \propto T$. As a result, we can deduce that the perpendicular vibrations give rise to a shift in the maximum of the distance distribution $\rho(r)$ with temperature, which corresponds, according to Equation (18), to a shift of the minimum of the effective pair potential $V(r)$. Accordingly, we can conclude that even in a simple harmonic crystal, the position of the effective pair potential (and so the true bond distance) changes with temperature due to the presence of transverse vibrations and therefore Equation (24) has to be replaced by the expression:

$$
\Delta C_{1}{ }^{*}=\frac{C_{3}{ }^{*}}{2 C_{2}{ }^{*}}+\Delta r_{\max }
$$

to include the shift with temperature of the effective pair potential. The bond thermal expansion $\Delta C_{1}{ }^{*}$ is thus given by the sum of two contributions: one from the asymmetry of the effective pair potential $V(r)$ and the other related to its rigid shift with temperature. Equation (30) has been tested by molecular dynamics simulations $^{[38,43]}$ and it seems to work satisfactorily [Figure 6]. However, its connection with Equation (14), rewritten as:

$$
\Delta C_{1}{ }^{*} \cong \Delta R+\frac{\Delta\left\langle\Delta u_{\perp}{ }^{2}\right\rangle}{2 R}
$$

remains not entirely clear. Indeed, if the shift of the effective pair potential $\Delta r_{\max }$ is uniquely due to the transverse vibrations, the thermal expansion $\Delta R$ of the apparent bond distance (to understand the one measured by diffraction) should correspond to the quantity $C_{3}{ }^{*} / 2 C_{2}{ }^{*}$. However, to the best of our knowledge, this correspondence was only observed for the first shell of germanium ${ }^{[37]}$, while for its outer shells and for other compounds ${ }^{[32,36]}$, the agreement completely fails, as shown in Figure 6 . This is likely due to the fact that the shape of the effective pair potential $V(r)$ is not temperature independent and therefore Equations (19)-(22) lose their validity. Furthermore, the relationship between Equations (30) and (31) is based on the fact that the shift of $V(r)$ with temperature is completely due to the perpendicular vibrations, but in a real crystal, there might be another contribution due to the crystal potential. For example, a negative shift of $V(r)$ was observed in $\mathrm{CuBr}$ and $\mathrm{AgI}$, tentatively explained in terms of ionic mobility ${ }^{[45,46]}$, and in $\mathrm{CdS} \mathrm{e}^{[42,43]}$. In addition, more refined treatments with a quantum approach do not lead to better results ${ }^{[47,48]}$. Although many works have been devoted to the study of the relationship between the effective pair potential and crystal potential ${ }^{[49-53]}$, this problem remains open and is not simple to solve. 


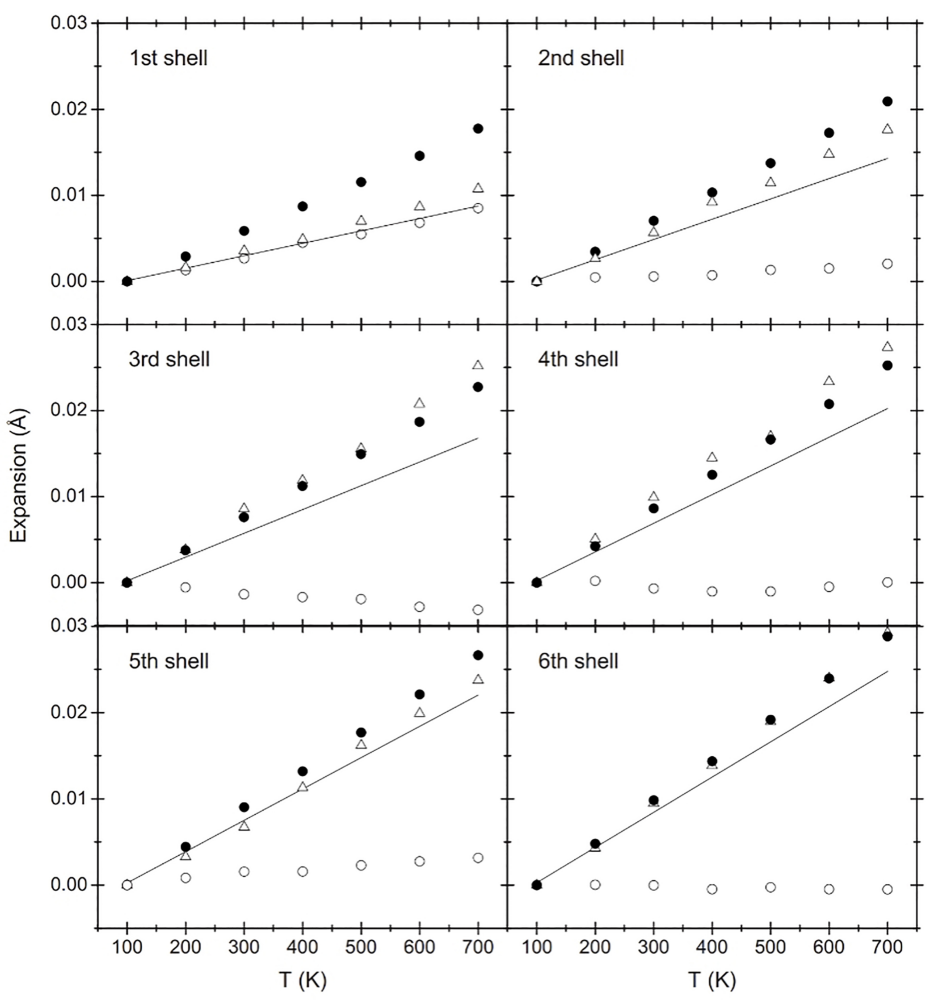

Figure 6. Comparison between bond thermal expansions $\Delta C_{1}{ }^{*}$ (solid circles) and the contributions due to the asymmetry $C_{3}{ }^{*} / 2 C_{2}{ }^{*}$ (open circles) and to the shift $\Delta r_{\max }$ (triangles) of the effective pair potential for the first six coordination shells of germanium. Solid lines are the crystallographic thermal expansions $\Delta R$ reported for comparison. It is evident, in good approximation, that $\Delta C_{1}{ }^{*}=C_{3}{ }^{*} / 2 C_{2}{ }^{*}+\Delta r_{\max }$. In

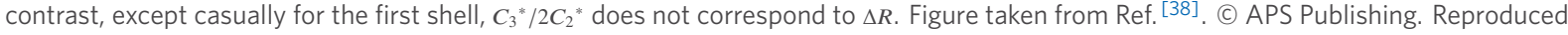
with permission. All rights reserved.

Finally, as a last observation, according to Equation (28), the transverse vibrations also produce an intrinsic asymmetry (here with $C_{3}{ }^{*} \propto T^{3}$ ) of the distance distribution $\rho(r)$, which is independent of the crystal anharmonicity. However, because the average position $C_{1}{ }^{*}$ and the maximum distribution position $r_{\max }$ coincide with each other, we can infer that such intrinsic asymmetry can be neglected. This claim has been validated by numerical simulations, as well as for high values of anisotropy ${ }^{[30]}$. Consequently, it can be concluded that, in general, the third cumulant of EXAFS is mostly related to the real anharmonicity of the crystal potential.

\section{DATA ANALYSIS STRATEGY}

The aim of this section is to provide information regarding the data analysis procedure to obtain accurate EXAFS results for studying the local dynamics. It is assumed that the reader has knowledge of the standard EXAFS analysis procedure.

Equation (7) is employed to best fit the EXAFS signal and to determine the cumulants $v s$. temperature of a given coordination shell. In this equation, the scattering-path amplitude and phase shift, $f(k)$ and $\varphi(k)$, respectively, are calculated by ab initio methods, typically using the FEFF code ${ }^{[54,55]}$. This step can be avoided by utilizing the "ratio method" to analyze the data of the first shell where multiple scattering can be neglected ${ }^{\text {[29] }}$. The photoelectron mean free path $\lambda(k)$ is also calculated. The free fit parameters are therefore the EXAFS cumulants, the loss factor $S_{0}{ }^{2}$ (the coordination number $N$ is known for crystals) and the binding energy $E_{0}$ of the core electron. 
The latter two terms have to be handled with careful attention. Indeed, $E_{0}$ and $S_{0}{ }^{2}$ are strongly correlated with the odd and even cumulants, respectively, with the result that the value of $E_{0}$ strongly affects the absolute value of $C_{1}{ }^{*}$ (and $C_{3}{ }^{*}$ ), while the value of $S_{0}{ }^{2}$ strongly affects the absolute value of $C_{2}{ }^{*}$ (and $C_{4}{ }^{*}$ ). Typically, a shift of $1 \mathrm{eV}$ in $E_{0}$ changes the value of $C_{1}{ }^{*}$ by $\sim 0.01 \AA$. Accordingly, for the present purposes, we cannot trust the "absolute" values of the EXAFS cumulants obtained through the best-fitting procedure, but we must consider the "relative" values in accordance with the following procedure. Let us mention here that a resolution better than $10 \mathrm{fm}$ was reached by EXAFS on the relative difference of nearest-neighbor interatomic distances of ${ }^{70} \mathrm{Ge}$ and ${ }^{76} \mathrm{Ge}$ isotopes ${ }^{[56,57]}$, proving the significant potential of EXAFS in the study of relative differences.

Firstly, all the experimental spectra have to be aligned in energy before the extraction of the EXAFS signals. This can be achieved through the reference spectra collected during the experiment, or, in the absence of these, through the spectrum at the lowest temperature, which can be used as reference. Obviously, in this second case, we must ensure that there is no shift in the edge position due to valence changes with temperature ${ }^{\text {[27] }}$. The error in the spectral alignment should not exceed $0.1 \mathrm{eV}$. The EXAFS signals can now be extracted by taking care to adopt the same process for all spectra.

Secondly, since there is no reason that $E_{0}$ and $S_{0}^{2}$ should vary with temperature, their values must be fixed during the best-fitting procedure. The most reasonable choice is to perform a first trial analysis where $E_{0}$ and $S_{0}{ }^{2}$ are left free and then set them to their average values. Fixing $E_{0}$ and $S_{0}{ }^{2}$ is essential to remove the effects of correlation on the relative values of EXAFS cumulants and to obtain more regular trends. Figure 7 shows an example of the best-fit results obtained with $E_{0}$ and $S_{0}{ }^{2}$ left free and then with $E_{0}$ and $S_{0}{ }^{2}$ fixed to their average values. Note the strong correlation between $\Delta C_{1}{ }^{*}$ and $E_{0}$ and between $\Delta C_{2}{ }^{*}$ and $S_{0}{ }^{2}$. Similar correlations can also be found for $\Delta C_{3}{ }^{*}$ and $\Delta C_{4}{ }^{*}$ (not shown here), although less important. As reported in Figures $7 \mathrm{~A}$ and $\mathrm{C}$, shifts toward positive (or negative) values of $E_{0}$ produce an increase (or decrease) of $\Delta C_{1}{ }^{*}$. Analogously, an increase (or decrease) of $S_{0}{ }^{2}$ produces a growth (or reduction) of $\Delta C_{2}{ }^{*}$ to compensate for the over/underestimation of the EXAFS amplitude due to $S_{0}{ }^{2}$. This is clearly evident from Figures $7 \mathrm{~B}$ and D. Fixing $E_{0}$ and $S_{0}{ }^{2}$ is therefore the key factor to obtain more regular trends for the EXAFS cumulants.

Thirdly, EXAFS cumulants higher than the second order must be considered in the analysis when possible. Indeed, the third and fourth cumulants have to be used to include anharmonicity and to thus obtain accurate values of the first and second cumulants, respectively ${ }^{[44,46,58]}$. This is particularly true for the interatomic distance expansion $\left(\Delta C_{1}{ }^{*}\right)$, where, by neglecting the third cumulant $C_{3}{ }^{*}$ (i.e., the Gaussian approximation), it is easy to find wrong values for $\Delta C_{1}{ }^{*}$. As an example, Figure 8 shows the Sc-F bond expansion found in scandium fluoride by including or excluding $C_{3}{ }^{*}$. It can be noted that the third cumulant, if neglected, leads to an underestimation of $\Delta C_{1}{ }^{*}$. On the other hand, it is understandable that by fitting an asymmetric distribution to the right using a Gaussian distribution, we obtain an underestimation of the average distance. Numerical estimates were performed to determine the effects of neglecting higher order cumulants in EXAFS analysis ${ }^{[59]}$. Neglection of the third cumulant leads to an underestimation of $\Delta C_{1}{ }^{*}$ when $\Delta C_{3}{ }^{*}>0$ and an overestimation when $\Delta C_{3}{ }^{*}<0$. Neglection of the fourth cumulant gives an underestimation of $\Delta C_{2}{ }^{*}$ when $\Delta C_{4}{ }^{*}>0$ and an overestimation when $\Delta C_{4}{ }^{*}<0$. Therefore, the third and fourth cumulants should be included in the EXAFS analysis. However, in some cases, inclusion of the third and fourth cumulants can significantly worsen the temperature trend of $\Delta C_{1}{ }^{*}$ and $\Delta C_{2}{ }^{*}$, respectively. Moreover, their inclusion seems to be important for the first coordination shell but much less important for the outer shells, where the correlation of atomic motion and anharmonicity are typically much lower than the first shell ${ }^{[38,43,60,61]}$. This can be seen, for example, in Figure 6, where the anharmonic contribution $C_{3}{ }^{*} / 2 C_{2}{ }^{*}$ is smaller for the outer shells than for the first one.

Finally, once the relative values $\Delta C_{1}{ }^{*}$ and $\Delta C_{2}{ }^{*}$ are determined, we must proceed to find the absolute value of the parallel and perpendicular MSRDs, $\left\langle\Delta u_{\|}{ }^{2}\right\rangle$ and $\left\langle\Delta u_{\perp}{ }^{2}\right\rangle$. In the Born-von Karman (harmonic) approxima- 

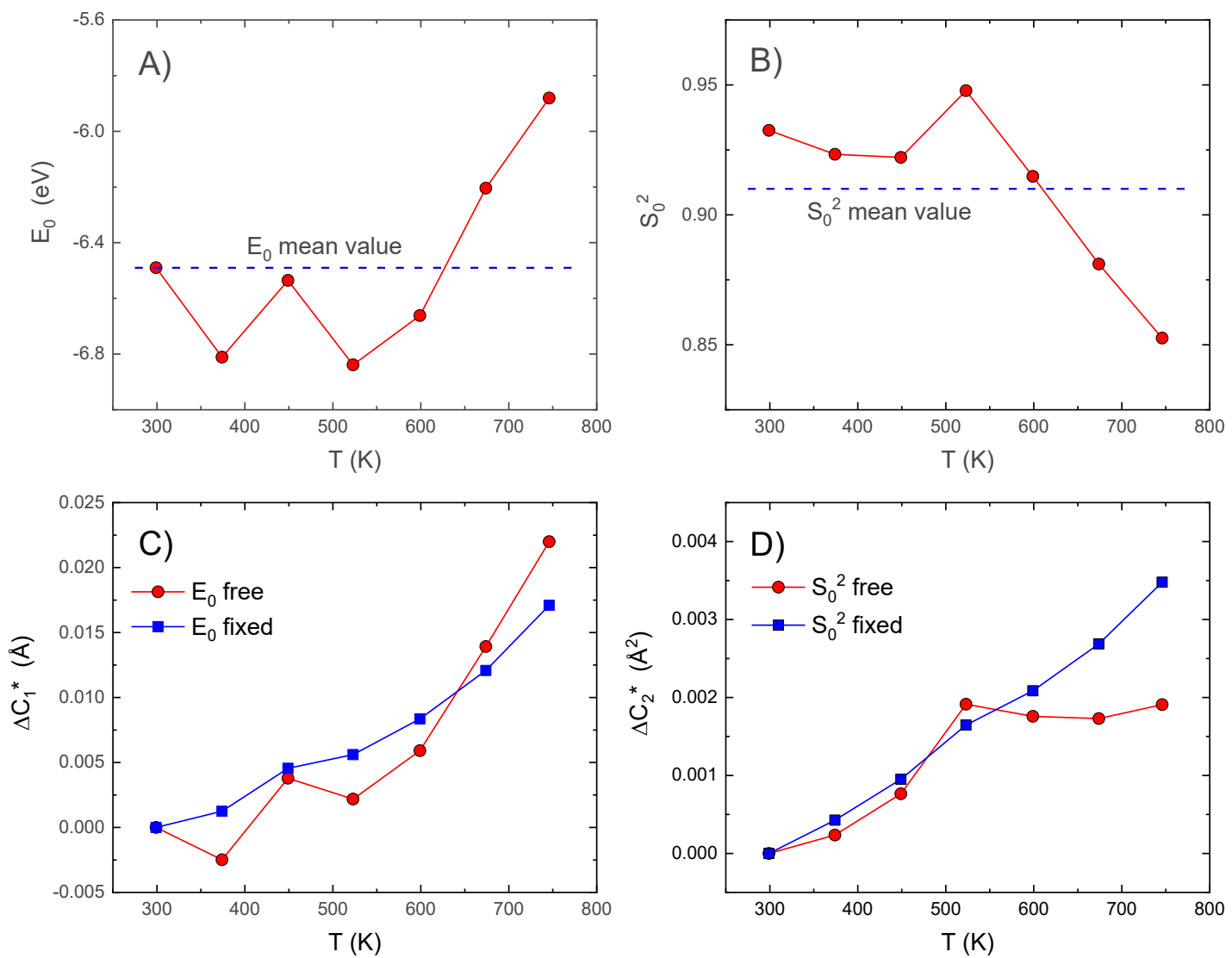

Figure 7. EXAFS best-fit results obtained for the first Sc-F shell of scandium fluoride[65]. Panels $A$ and B show, respectively, the temperature behavior of $E_{0}$ and $S_{0}{ }^{2}$ left free (red circles). Panels $C$ and D show, respectively, the temperature behavior of $\Delta C_{1}{ }^{*}$ and $\Delta C_{2}{ }^{*}$ with $E_{0}$ and $S_{0}{ }^{2}$ left free (red circles) and with $E_{0}$ and $S_{0}{ }^{2}$ fixed to their average value (blue squares), here $-6.49 \mathrm{eV}$ for $E_{0}$ and 0.91 for $S_{0}{ }^{2}$. Lines are a guide to the eyes. Note the strong correlation between $\Delta C_{1}{ }^{*}$ and $E_{0}$, and between $\Delta C_{2}{ }^{*}$ and $S_{0}{ }^{2}$. Accordingly, when $E_{0}$ and $S_{0}{ }^{2}$ are fixed, a more regular trend is obtained for $\Delta C_{1}{ }^{*}$ and $\Delta C_{2}{ }^{*}$.

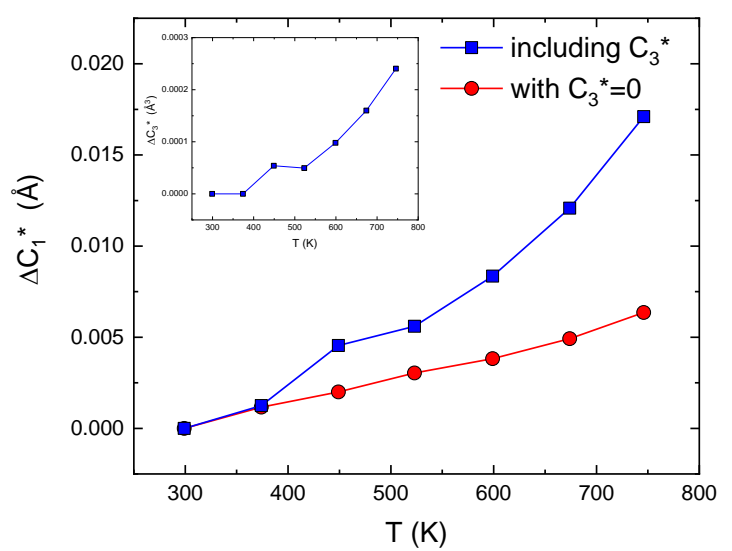

Figure 8. Bond thermal expansion $\Delta C_{1}{ }^{*}$ for the first shell of scandium fluoride obtained by including (blue squares) or excluding (red circles) the third cumulant $C_{3}{ }^{*}$. In the present case, by neglecting the third cumulant, $\Delta C_{1}{ }^{*}$ is widely underestimated. The inset shows the temperature behavior of $\Delta C_{3}{ }^{*}$.

tion, Equation (16) for the parallel MSRD at a given temperature $T$ can be written as ${ }^{[62]}$ :

$$
\left\langle\Delta u_{\|}{ }^{2}\right\rangle=\frac{1}{N} \sum_{\lambda, \bar{q}} \frac{h}{8 \pi^{2} v(\lambda, \bar{q})} \operatorname{coth}\left(\frac{h v(\lambda, \bar{q})}{2 k_{B} T}\right)\left|\left(\frac{\bar{w}_{1}(\lambda, \bar{q}) e^{i \bar{q} \cdot \bar{R}}}{\sqrt{m_{1}}}-\frac{\bar{w}_{0}(\lambda, \bar{q})}{\sqrt{m_{0}}}\right) \cdot \hat{R}\right|^{2}
$$




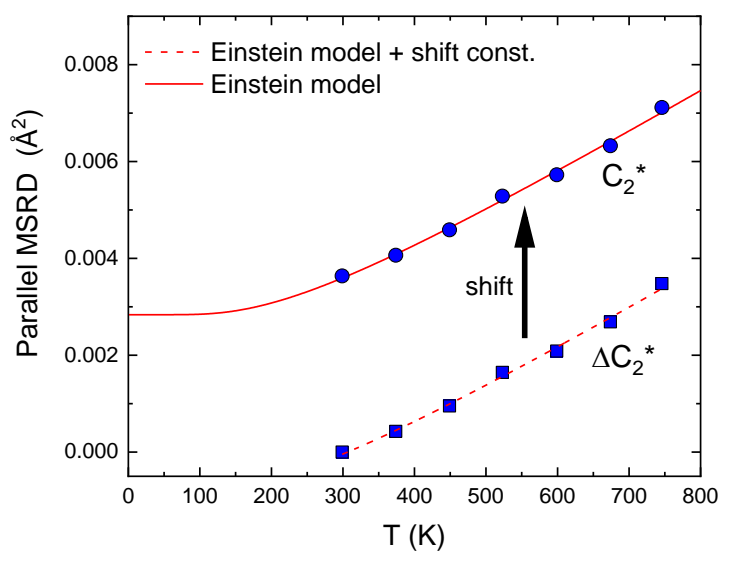

Figure 9. Temperature dependence of $\Delta C_{2}{ }^{*}$ (squares) and best fit using the correlated Einstein model (dashed line), which gives $v_{\|}=13.1$ $\mathrm{THz}$ and a constant shift $\left|a_{\|}\right|=0.0036 \AA^{2}$. Circles and solid line are, respectively, the resulting parallel MSRD and the corresponding Einstein model. Error bars have been omitted for clarity.

where $m_{0}$ and $m_{1}$ are the masses of the absorber and backscatterer atoms, respectively, $\bar{w}_{0}(\lambda, \bar{q})$ and $\bar{w}_{1}(\lambda, \bar{q})$ are their eigenvectors corresponding to the vibrational mode $(\lambda, \bar{q})$ of the Brillouin zone, $v(\lambda, \bar{q})$ is the vibrational frequency, $\hat{R}$ is the bond direction and $N$ is the number of unit cells. The sum is over all normal modes of the Brillouin zone, weighted by the Bose-Einstein population factor $\operatorname{coth}\left(\frac{h v(\lambda, \bar{q})}{2 k_{B} T}\right) / v(\lambda, \bar{q})$.

Equation (32) describes the thermal motion including the zero-point energy at $0 \mathrm{~K}$ and it can be used as the starting point to derive phenomenological models to account for the temperature dependence of the parallel MSRD. The first one is the correlated Debye model where only acoustic branches with linear dispersion are considered and the Brillouin zone is substituted by a Debye sphere ${ }^{[63,64]}$. An alternative model that is easier to use is the correlated Einstein model, which considers three-dimensional quantum harmonic oscillators with the same frequency $v_{\|}{ }^{[49,64]}$. With this simple assumption, Equation (32) becomes:

$$
\left\langle\Delta u_{\|}^{2}\right\rangle=\frac{h}{8 \pi^{2} \mu v_{\|}} \operatorname{coth}\left(\frac{h v_{\|}}{2 k_{B} T}\right)
$$

where $\mu=m_{0} m_{1} /\left(m_{0}+m_{1}\right)$ is the reduced mass of the pair of atoms. The Einstein model only depends on one parameter, the frequency $v_{\|}$, which is different for different coordination shells. The Einstein frequency $v_{\|}$can, in turn, be connected to a bond-stretching effective force constant, $k_{\|}=4 \pi^{2} \mu v_{\|}^{2}$, which represents a measure of the strength of the bond.

The Einstein model Equation (33) can be utilized to recover the absolute values of the parallel MSRD, i.e., $C_{2}{ }^{*}$, by fitting the temperature dependence of $\Delta C_{2}{ }^{*}$. Figure 9 shows an example of this fit for the Sc-F distance measured in scandium fluoride ${ }^{[65]} . \Delta C_{2}{ }^{*}$ is fitted by the Einstein model plus a negative shift constant " $a_{\|}$", so that the fitting parameters are only $v_{\|}$and $a_{\|}$. The absolute value of $C_{2}{ }^{*}$, corresponding to $\left\langle\Delta u_{\|}{ }^{2}\right\rangle$, is then given by $\Delta C_{2}{ }^{*}+\left|a_{\|}\right|$.

Similarly, the Einstein model can also be used to determine the absolute values of the perpendicular MSRD by fitting the temperature dependence of $\Delta\left\langle\Delta u_{\perp}{ }^{2}\right\rangle$, recovered by inverting Equation (31). Since the perpendicular MSRD is a projection of relative atomic motion onto a plane, while the parallel MSRD is a projection along the bond direction, the Einstein model for $\left\langle\Delta u_{\perp}{ }^{2}\right\rangle$ differs from that for $\left\langle\Delta u_{\|}{ }^{2}\right\rangle$ by a factor of two ${ }^{[64]}$ :

$$
\left\langle\Delta u_{\perp}^{2}\right\rangle=\frac{h}{4 \pi^{2} \mu v_{\perp}} \operatorname{coth}\left(\frac{h v_{\perp}}{2 k_{B} T}\right)
$$

Figure 10A shows an example comparison between the true bond expansion $\Delta C_{1}{ }^{*}$ measured by EXAFS (for 

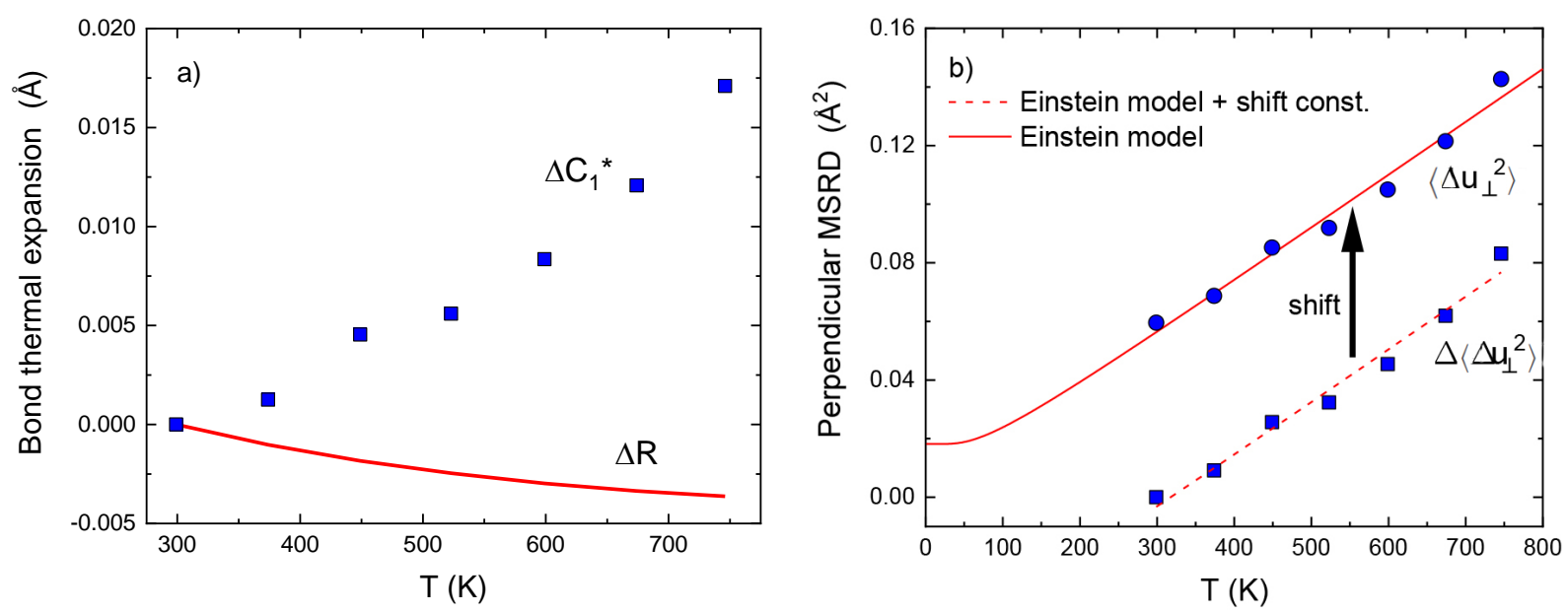

Figure 10. (A) Comparison between true bond expansion $\Delta C_{1}{ }^{*}$ measured by EXAFS (squares) and apparent bond expansion $\Delta R$ measured by diffraction (solid line). (B) Resulting temperature dependence of $\Delta\left\langle\Delta u_{\perp}{ }^{2}\right\rangle$ (squares) and best fit using the correlated Einstein model (dashed line), which gives $v_{\perp}=4.1 \mathrm{THz}$ and a constant shift $\left|a_{\perp}\right|=0.060 \AA^{2}$. Circles and solid line are, respectively, the resulting perpendicular MSRD and the corresponding Einstein model. Error bars have been omitted for clarity.

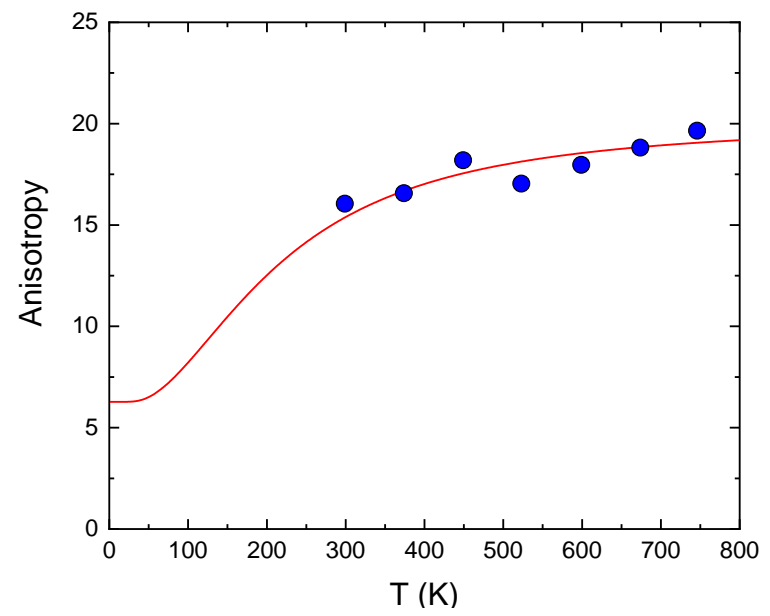

Figure 11. Resulting anisotropy of the relative thermal vibrations, $\gamma=\left\langle\Delta u_{\perp}{ }^{2}\right\rangle /\left\langle\Delta u_{\|}{ }^{2}\right\rangle$ : experimental data (circles) and corresponding best-fit Einstein model (solid line). Error bars have been omitted for clarity.

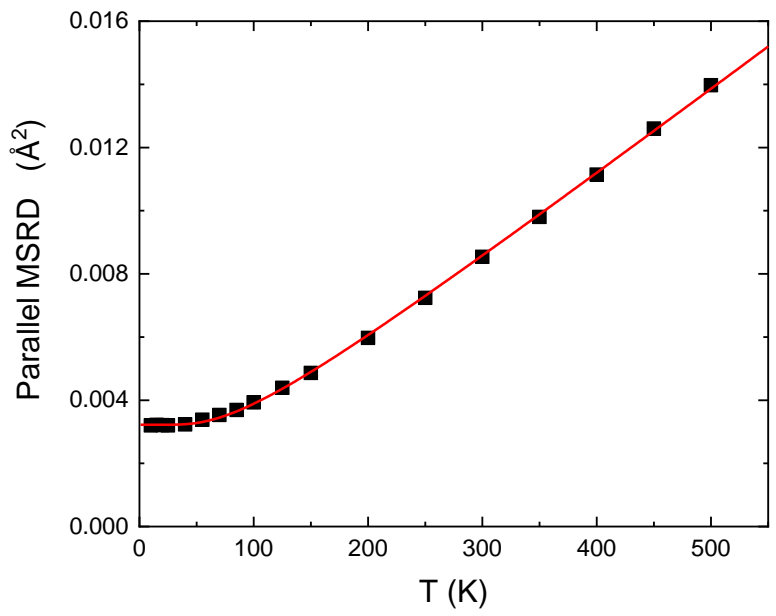

Figure 12. Parallel MSRD measured by EXAFS for the first $\mathrm{Cu}-\mathrm{Cu}$ shell of copper (squares) and corresponding best-fit Einstein model (solid line) ${ }^{[36]}$. Despite its simplicity, the Einstein model satisfactorily reproduces the temperature behavior of MSRDs.

the Sc-F bond of scandium fluoride) and the apparent bond expansion $\Delta R$ measured by Bragg diffraction, from which $\Delta\left\langle\Delta u_{\perp}{ }^{2}\right\rangle$ is obtained by inverting Equation (31), as reported in Figure 10B. By fitting $\Delta\left\langle\Delta u_{\perp}{ }^{2}\right\rangle$ through the correlated Einstein model [Equation (34)] plus a negative shift constant " $a_{\perp}$ ", the absolute value of the perpendicular MSRD, $\left\langle\Delta u_{\perp}{ }^{2}\right\rangle$, can be obtained by the sum $\Delta\left\langle\Delta u_{\perp}{ }^{2}\right\rangle+\left|a_{\perp}\right|$ [Figure 10B] and the resulting Einstein frequency $v_{\perp}$ can be related to a bond-bending effective force constant, $k_{\perp}=4 \pi^{2} \mu v_{\perp}{ }^{2}$. Once both $\left\langle\Delta u_{\|}{ }^{2}\right\rangle$ and $\left\langle\Delta u_{\perp}{ }^{2}\right\rangle$ are known, we can finally calculate the anisotropy of the relative thermal vibrations by the ratio $\gamma=\left\langle\Delta u_{\perp}{ }^{2}\right\rangle /\left\langle\Delta u_{\|}{ }^{2}\right\rangle$, as shown in Figure 11. In this example, large values of $\gamma$ are found, thus indicating that the transverse thermal vibrations of Sc-F are extremely large in scandium fluoride, much more than the longitudinal ones, as predicted by first-principles calculations ${ }^{[6]}$.

Although the correlated Einstein model is only a simple phenomenological model, it has the ability to reproduce satisfactorily the temperature behavior of $\left\langle\Delta u_{\|}{ }^{2}\right\rangle$ and $\left\langle\Delta u_{\perp}{ }^{2}\right\rangle$. As an example, Figure 12 shows how 
the Einstein model excellently reproduces the parallel MSRD of the first $\mathrm{Cu}-\mathrm{Cu}$ shell of copper in the whole temperature range. The discrepancy between the MSRDs calculated from lattice dynamics and derived from the best-fitting Einstein model has been evaluated for different crystalline structures, with the error typically found to be of the order of a few percent, in particular at low temperatures ${ }^{[67]}$. This makes the Einstein model the most used model in this type of EXAFS analysis and is preferable to other models, such as the Debye model, due to its simplicity.

\section{APPLICATIONS}

In this section, examples of studies are provided that show the capability of EXAFS in the study of local dynamics. Obviously, good quality EXAFS data are required to obtain reliable information on the local vibrational dynamics. This is particularly true for the perpendicular MSRD, which is far more complicated to obtain than the parallel MSRD.

\subsection{EXAFS as a benchmark for theoretical calculations}

It is obvious that the local dynamics information obtained by EXAFS represents an excellent benchmark for theoretical calculations, because the EXAFS MSRDs depend not only on the frequency of the vibrational modes, but also on their eigenvectors. This is in addition to the EXAFS cumulants depending on the effective pair potential that is related to the real three-dimensional crystal potential. For example, EXAFS was recently used by Kuzmin and co-workers ${ }^{[68-70]}$ to test the accuracy of several force-field models employed in molecular dynamics simulations. Force-field models that give lattice parameters, bulk moduli and elastic constants in good agreement with experimental data can still fail to reproduce the temperature dependence of EXAFS data. Thus, EXAFS provides additional information that enables discrimination between different models.

Various theoretical calculations have been carried out to predict EXAFS parameters and to validate interatomic potential models. The temperature dependence of the EXAFS cumulants up to the third or fourth order was calculated by Yokoyama et al. ${ }^{[71]}$ for a $\mathrm{Br}_{2}$ molecule, solid krypton and nickel in the path-integral framework. Excellent agreement was obtained with the experimental cumulants by using the effective-potential method. Path-integral Monte Carlo calculations of EXAFS cumulants were also performed by Beccara et al. ${ }^{[72]}$ for fcc copper and the sensitivity to the choice of the interaction potential was tested, indicating the need to adopt a multi-body potential to satisfactorily reproduce the experimental EXAFS third cumulant [Figure 13]. Vila et al. ${ }^{[52]}$ introduced an $a b$ initio equation of motion method to calculate the temperature dependence of the MSRDs of EXAFS and successfully it applied to germanium, $\mathrm{Zn}^{2+}$-tetraimidazole and zirconium tungstate. Many other works have combined EXAFS and molecular dynamics to study the structural and dynamic properties of crystals ${ }^{[73-78]}$, nanoparticles ${ }^{[79-81]}$, glasses ${ }^{[82-84]}$ and liquids ${ }^{[85-87]}$.

\subsection{Nanoparticles}

Detailed EXAFS studies have been conducted to explore the local dynamics of nanoparticle systems. In one of the earliest studies, Rockenberger et al. ${ }^{[88]}$ investigated the size dependence of the structural and dynamic properties of CdS nanoparticles in a size range from 1.3 to $12 \mathrm{~nm}$. They observed that these properties are related to the surface-to-volume ratio and to the method of surface stabilization of the nanoparticles. A temperatureindependent static contribution to the MSRD of the Cd-S bonds was identified. This static contribution is strongly size dependent. In contrast, the vibrational amplitudes of the Cd-S bonds are slightly reduced with decreasing particle size. The asymmetry of the interatomic Cd-S pair distribution, evaluated through the third EXAFS cumulant, is increased with reducing particle size and shows the possibility of distinguishing the crystal structure of CdS nanoparticles.

A decade later, EXAFS was used by Araujo et al. ${ }^{[89]}$ to study the vibrational properties of Ge nanocrystals (NCs). The EXAFS Debye-Waller factor revealed that the Ge-Ge bonds of Ge NCs are stiffer than both bulk 


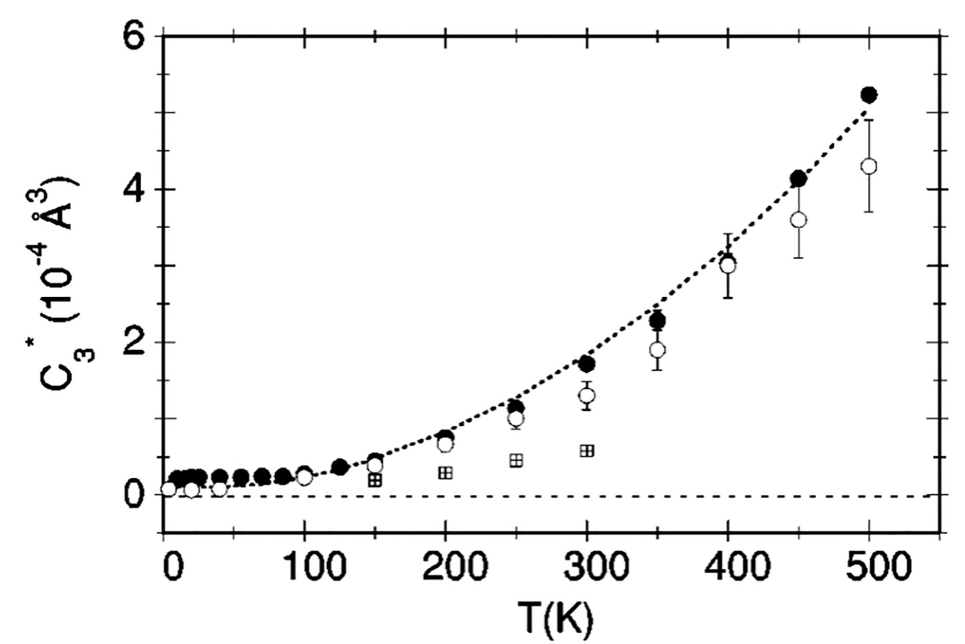

Figure 13. Third EXAFS cumulant for the first shell of fcc copper: from EXAFS data (full circles) and from path-integral Monte Carlo calculations with a many-body potential (open circles) and a two-body potential (crossed squares). The dashed line is the best-fitting model of experimental data. Figure taken from Ref. ${ }^{[72]}$. (c) APS Publishing. Reproduced with permission. All rights reserved.

crystalline and amorphous Ge, with a static contribution that lies between theirs [Figure 14]. The higher static disorder in Ge NCs with respect to the crystal was attributed to the presence of undercoordinated atoms on the surface and the resulting internal strain. Moreover, the bond thermal expansion obtained for Ge NCs is smaller than for crystalline Ge, in good agreement with existing data for other nanocrystalline semiconductors ${ }^{[90,91]}$. Somewhat different behavior was observed by Sprouster et al. ${ }^{[92]}$, who investigated the size-dependent vibrational properties of Co nanoparticles by temperature-dependent EXAFS. Compared to bulk Co, the authors noted a softening of the Co-Co bonds in the smallest nanoparticles, attributed to the strong influence of undercoordinated bonds, as well as reductions in the average bond length and coordination number. Enhanced structural disorder and asymmetrical deviation from a Gaussian-like interatomic distance distribution with decreasing particle size were confirmed.

Furthermore, the vibrational properties of gold nanoparticles have also been subject to EXAFS studies. Comaschi et al. ${ }^{[93]}$ studied the thermal behavior of Au nanoparticles with sizes ranging from 2.4 to $5.0 \mathrm{~nm}$. With respect to the bulk crystal counterpart, the authors observed for the first four shells a progressive reduction of the average bond length and coordination number with decreasing particle size, as well as a simultaneous increase in the static disorder. More interestingly, a reduction of the $\mathrm{Au}-\mathrm{Au}$ bond thermal expansion was found, with a curious crossover from an initial thermal expansion to a thermal contraction in the smallest particles, tentatively interpreted as being due to the presence of discrete electronic energy levels induced by the finite size particles ${ }^{[94]}$. Duan et al. ${ }^{[95]}$ confirmed the contraction of the average Au-Au bond length, the decrease of coordination number and the presence of static disorder in Au147 nanoparticles compared to the bulk material. However, they were able to observe a bond expansion for the surface-surface bonds, accompanied by a softening of the vibrational modes, while the surface-core and core-core bonds contracted. A recent review by Timoshenko et al. ${ }^{[96]}$ reports the latest advancements in the study of the structure and dynamics of metal nanoparticles by combining EXAFS spectroscopy and atomistic simulations.

A noteworthy EXAFS study of the local dynamics of nanoparticles was carried out by Hu et al. ${ }^{[97]}$ for scandium fluoride. Static disorder and a decrease in the average bond length were observed for both the Sc-F and ScSc distances with respect to the bulk counterpart. However, the most important result to be noted was the large stiffening of the average Sc-F bond bending, which resulted in a reduction of the Sc-F anisotropy and negative thermal expansion (NTE). The use of EXAFS spectroscopy to investigate NTE materials represents one of the most successful fields of EXAFS applications for studying vibrational dynamics, as described in the 


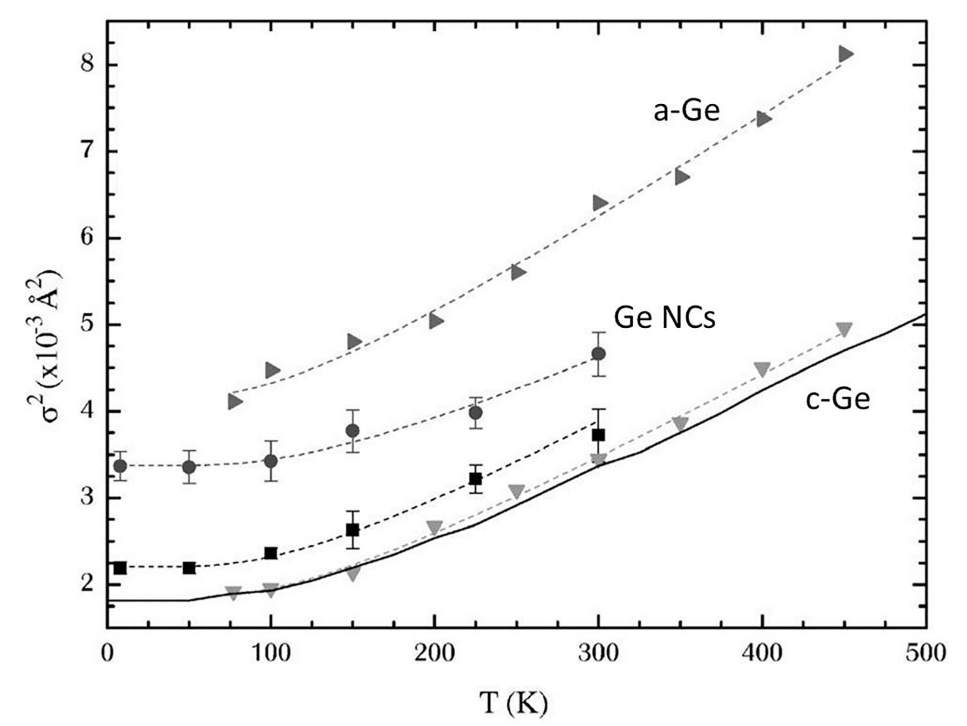

Figure 14. EXAFS Debye-Waller factor measured as a function of temperature in Ge nanocrystals (circles), bulk crystalline Ge (squares and down-triangles) and amorphous Ge (right-triangles), with the respective correlated Einstein model fits (dashed lines). The solid line is the $\mathrm{Ge}-\mathrm{Ge}$ dynamic contribution in c-Ge from ab initio calculations ${ }^{[138]}$. The reduced slope of Debye-Waller for Ge NCs means that the Ge-Ge bonds in Ge NCs are stiffer than crystalline and amorphous Ge. Moreover, Ge-Ge static disorder is observed in Ge NCs, lying between that of c-Ge and a-Ge. Figure taken from Ref. ${ }^{[89]}$. (c) APS Publishing. Reproduced with permission. All rights reserved.

next section.

\subsection{Negative thermal expansion materials}

Thermal expansion represents a problem for many materials and engineering applications and its control is a challenge for materials design. After the discovery of materials that exhibit strong NTE over wide temperature ranges ${ }^{[98,99]}$, the interest in NTE has rapidly expanded and the prospect of controlling thermal expansion has become possible. Although it is known that NTE arises from a range of different mechanisms ${ }^{[100]}$, the general comprehension of NTE (and related phenomena) is still incomplete. In this regard, EXAFS spectroscopy is of great help since it provides unique information on the local vibrational dynamics. Like EXAFS, the neutron pair distribution function (PDF) is sensitive to the atom-atom correlation, but the overlap of neighboring peaks in PDF analysis makes it extremely difficult to investigate the local dynamics of nearest neighboring bonds with sufficient accuracy.

To the best of our knowledge, the first EXAFS studies to investigate the local dynamics of NTE materials were performed on zirconium tungstate ${ }^{[101,102]}$, the most popular NTE material, and cuprite structures ${ }^{[35,103,104]}$. For $\mathrm{ZrW}_{2} \mathrm{O}_{8}$, EXAFS was adopted to investigate the thermal displacements of $\mathrm{Zr}, \mathrm{W}$ and $\mathrm{O}$ atoms and the correlation in the motion of the $\mathrm{WO}_{4}$ tetrahedra and $\mathrm{ZrO}_{6}$ octahedra. The experimental results were used to tentatively explain the NTE mechanism of $\mathrm{ZrW}_{2} \mathrm{O}_{8}$. EXAFS analysis of cuprite structures $\mathrm{M}_{2} \mathrm{O}(\mathrm{M}=\mathrm{Cu}$ or $\mathrm{Ag}$ ) allowed the different thermal behavior of M-M pairs belonging to the same or different networks of $\mathrm{M}_{4} \mathrm{O}$ tetrahedra to be distinguished, with the first decreasing the interatomic distance with increasing temperature and the second expanding [Figure 15]. This experimental finding, together with the intense relative motion observed for the $\mathrm{M}-\mathrm{M}$ atomic pair along the edges of the $\mathrm{M}_{4} \mathrm{O}$ tetrahedra, rules out the possibility of explaining NTE in cuprite structures with a simple rigid unit mode model, because it is based on the phonon modes of rigid or quasi-rigid structural units ${ }^{[05]}$. As a result, a more flexible model, simply based on rigid nearestneighbor bonds and a tension effect, has been adopted to explain the NTE of cuprite structures ${ }^{[106]}$.

More recently, a number of crystals with the diamond-zincblende structure ( $\mathrm{Ge}, \mathrm{CuCl}, \mathrm{CdTe}, \mathrm{InP}$ and $\mathrm{GaAs}$ ), 

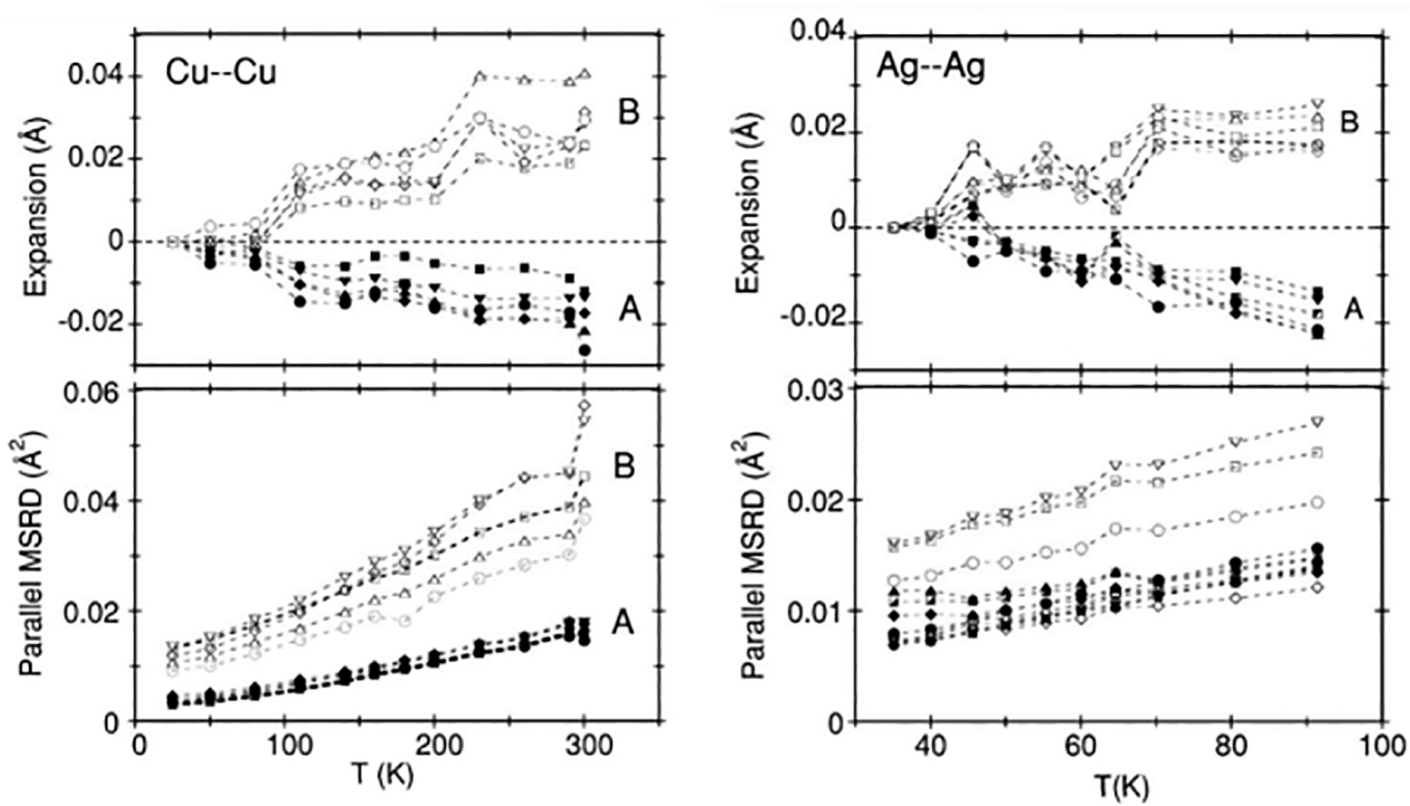

Figure 15. Bond thermal expansion (top panels) and parallel MSRDs (bottom panels) of the M-M second-shell distance in $\mathrm{Cu}_{2} \mathrm{O}$ (left panels) and $\mathrm{Ag}_{2} \mathrm{O}$ (right panels), for different constraints in the fitting procedure (for more details, see Ref. ${ }^{[35]}$ ). Closed and open symbols refer, respectively, to intra-tetrahedra (edges of $\mathrm{M}_{4} \mathrm{O}$ tetrahedra) and inter-tetrahedra M-M distances. The intra-tetrahedra contribution to NTE observed by EXAFS cannot be explained in terms of RUMs. Figure taken from Ref. ${ }^{[35]}$. (c) APS Publishing. Reproduced with permission. All rights reserved.

affected by NTE at low temperatures, have been investigated by EXAFS ${ }^{[21,32,33,37,107]}$. These studies showed that a correlation exists between the strength of NTE, the degree of bond ionicity and some of the parameters determined from EXAFS analysis, such as the anisotropy of the relative vibrations of nearest-neighbor pairs.

Direct experimental evidence of the NTE mechanism in $\mathrm{ScF}_{3}$, one of the most interesting materials with NTE properties, was possible as a result of EXAFS spectroscopy ${ }^{[65]}$. A positive expansion of the first-shell Sc-F bond distance and a concomitant shrinkage of the second-shell Sc-Sc distance were observed. This shows the existence of large vibrations of fluorine atoms perpendicular to the Sc-F-Sc linkage, indicating that the Sc-F bond is much softer to bend than to stretch, and that the relative motion of the Sc-Sc pair is more pronounced along the bond direction [Figure 16]. Although the latter effect might be too emphasized due to the large error bars in the Sc-Sc perpendicular MSRD, the combination of these findings indicates the presence of a "guitar-string" effect ${ }^{[108]}$ as the local mechanism responsible for NTE in $\mathrm{ScF}_{3}$. Soon after, EXAFS was used to investigate the isotropic zero thermal expansion of $\left(\mathrm{Sc}_{0.8} \mathrm{Fe}_{0.2}\right) \mathrm{F}_{3}$, where the $\mathrm{NTE}$ of $\mathrm{ScF}_{3}$ was suppressed by partially substituting $\mathrm{Sc}$ with $\mathrm{Fe}^{[109]}$. The EXAFS study of Purans et al. ${ }^{[10]}$ on $\mathrm{ReO}_{3}$, which is isostructural to $\mathrm{ScF}_{3}$, supports the concept that NTE is due to correlated movements of Re atoms in the transverse direction with respect to the-Re-O-Re-chains. Furthermore, the presence of some disordered rotations of the $\mathrm{ReO}_{6}$ octahedra explains why $\mathrm{ReO}_{3}$ shows a positive expansion from 150 to $\sim 500 \mathrm{~K}^{[110]}$.

EXAFS was also employed to study the local dynamics of Prussian blue analogues (PBAs), an important family of multifunctional materials with applications in catalysis, energy storage, magnetism, drug delivery and so on $^{[111]}$. In particular, some PBAs display NTE properties ${ }^{[12]}$. The chemical composition $\mathrm{MM}^{\prime}(\mathrm{CN})_{6}(\mathrm{M}=$ $\mathrm{Ga}, \mathrm{Fe}$, Ti or Sc; M' = Fe or Co), a cubic framework structure built by a cyanide-bridged network of octahedral units, was investigated by EXAFS. It was found that NTE is induced by the transverse vibrations of the atomic$\mathrm{M}-\mathrm{N} \equiv \mathrm{C}-\mathrm{M}$ '- linkages through a tension mechanism, since both $\mathrm{M}-\mathrm{N}$ and $\mathrm{M}$ '-C bonds are much softer to bend than to stretch. However, the main vibrational contribution to the NTE comes from the N atoms, since the 

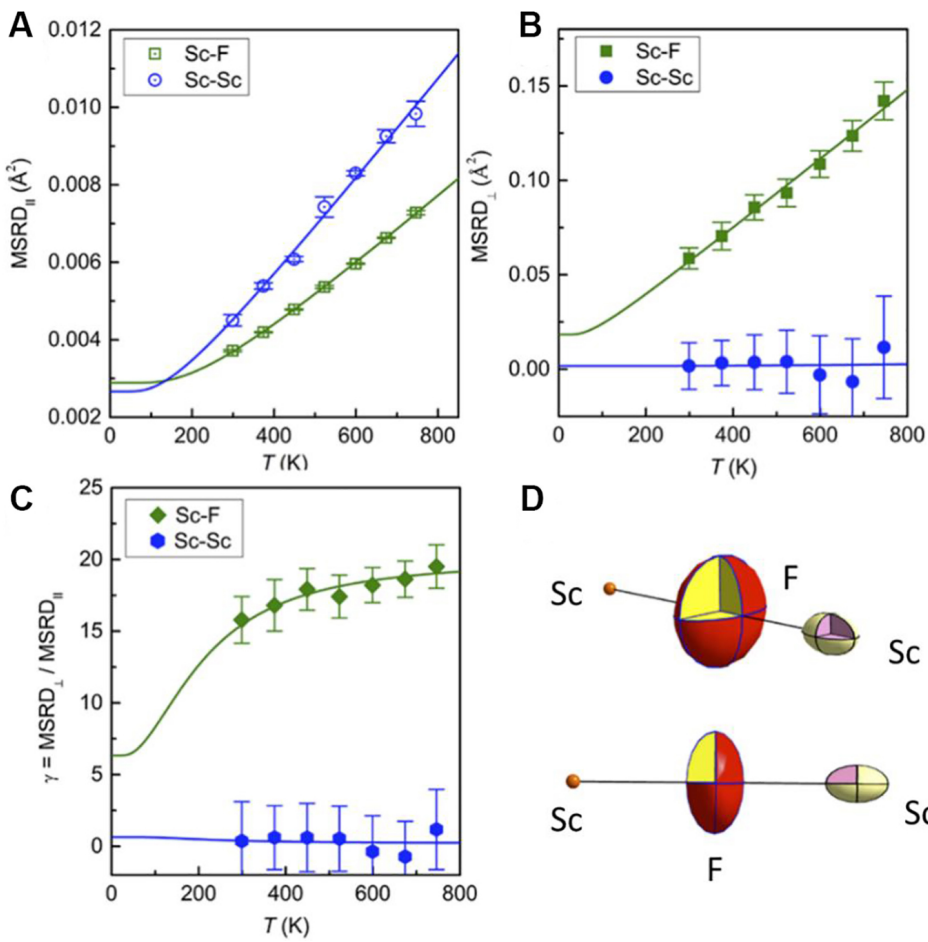

D
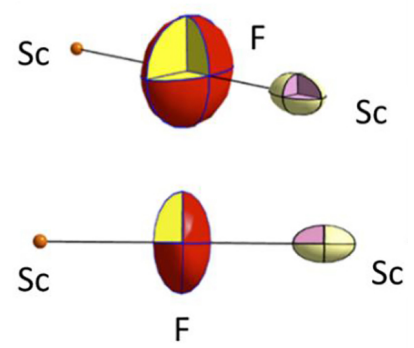

Figure 16. (A) Parallel MSRDs, (B) perpendicular MSRDs and (C) anisotropy of the relative thermal vibrations for the Sc-F and Sc-Sc atomic pairs determined by EXAFS in $\mathrm{ScF}_{3}$. The lines are the corresponding best-fit Einstein models. (D) Schematic representation of the thermal ellipsoids of the relative Sc-F and Sc-Sc vibrations reconstructed from EXAFS results. These results agree with a "guitar-string" effect as the local mechanism responsible for the NTE in $\mathrm{ScF}_{3}$. Figure taken from Ref. ${ }^{[65]}$. (c) ACS Publishing. Reproduced with permission. All rights reserved.

relative transverse vibrations of the M-N bonds are much larger than that of the $\mathrm{M}^{\prime}-\mathrm{C}$ bonds [Figure 17 ${ }^{[113-115]}$.

Very interesting is the fact that the insertion of guest ions or molecules in PBA structures can inhibit NTE, thereby opening the possibility of controlling the thermal expansion of materials. For examples, the incorporation of $\mathrm{K}^{+}$ions or $\mathrm{H}_{2} \mathrm{O}$ molecules in $\mathrm{YFe}(\mathrm{CN})_{6}$ switches its thermal expansion from strongly negative to strongly positive ${ }^{[116]}$, while the progressive insertion of $\mathrm{Na}^{+}$ions in $\mathrm{GaFe}(\mathrm{CN})_{6}$ changes the thermal expansion from negative to zero to positive ${ }^{[117]}$. In addition, the intercalation of $\mathrm{H}_{2} \mathrm{O}$ molecules in $\mathrm{TiCo}(\mathrm{CN})_{6}$ changes the thermal expansion from negative to zero below room temperature ${ }^{[118]}$. EXAFS allows us to understand the local mechanism at the origin of such thermal expansion behavior, i.e., the presence of guest ions or molecules hinders the transverse atomic vibrations, thus inhibiting the NTE. This effect is much more marked for the M-N bonds than for the M'-C bonds. This is clearly evident from Figure 18, where the EXAFS results for both $\mathrm{Na}: \mathrm{GaFe}(\mathrm{CN})_{6}$ and $\mathrm{TiCo}(\mathrm{CN})_{6}: \mathrm{H}_{2} \mathrm{O}$ are reported.

\subsection{Superconducting materials}

EXAFS was used to study superconducting materials. Lanzara et al. ${ }^{[19]}$ studied the local atomic correlations in the $\mathrm{HgBa}_{2} \mathrm{CuO}_{4+\delta}$ cuprate system across the superconducting transition temperature. The temperature dependence of the Debye-Waller factors for the $\mathrm{Cu}-\mathrm{O}$ and $\mathrm{Cu}$-Ba distances was determined and an anomalous decrease was observed for $\mathrm{Cu}-\mathrm{O}$ across the superconducting transition [Figure 19]. Through a comparative EXAFS-diffraction study, the atomic correlation coefficients of the $\mathrm{Cu}-\mathrm{O}$ and $\mathrm{Cu}-\mathrm{Ba}$ atomic pairs were estimated. A local rearrangement of $\mathrm{Cu}-\mathrm{O}$ correlations was observed to occur at the superconducting transition.

More recently, Joseph et al. ${ }^{[120]}$ studied the iron-oxypnictide superconductor system as a function of temperature. EXAFS showed a weak temperature and F-substitution dependence of the Fe-As bond length, in 

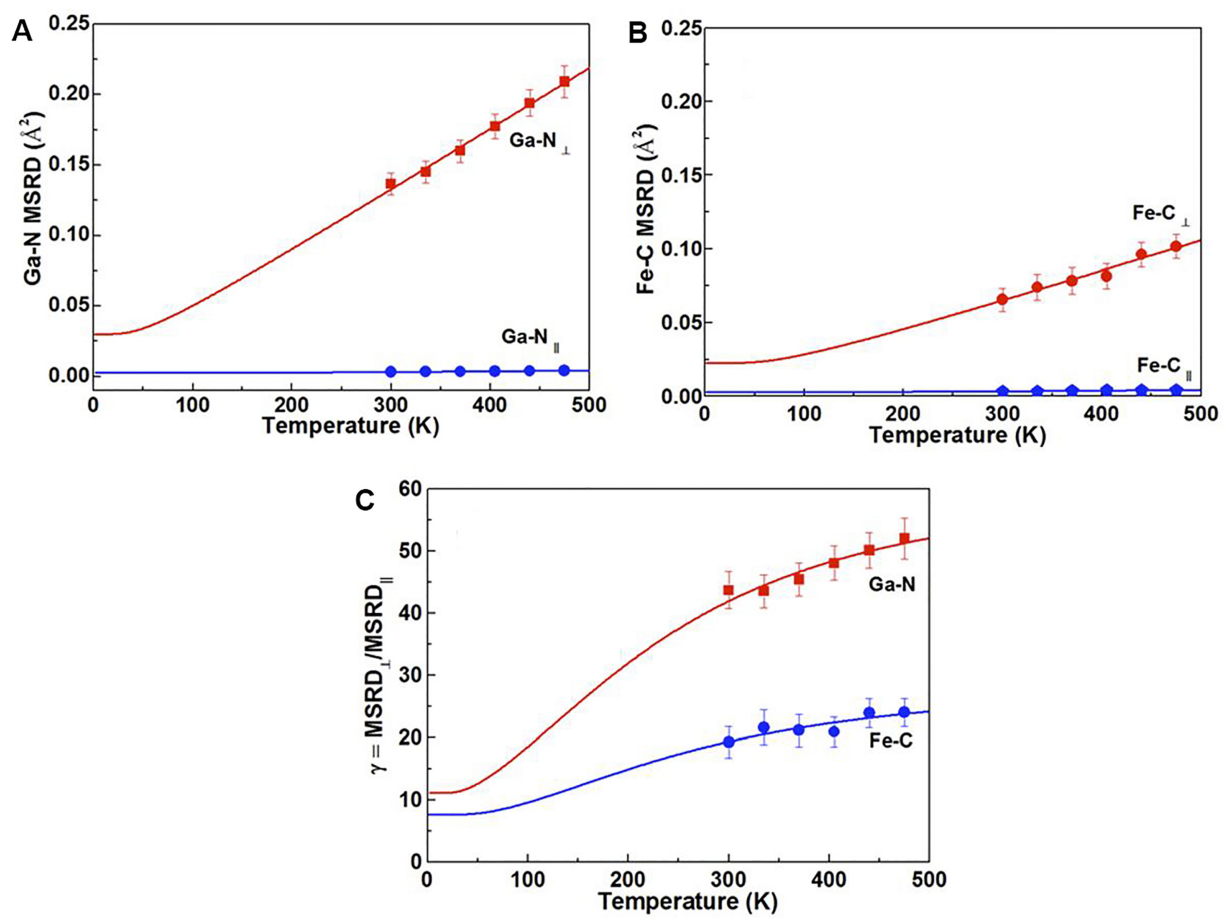

Figure 17. Parallel and perpendicular MSRDs for the Ga-N and Fe-C bonds [panels (A) and (B), respectively] of GaFe(CN) 6 . Panel (C) shows the resulting anisotropy of the relative thermal vibrations. Both $\mathrm{Ga}-\mathrm{N}$ and $\mathrm{Fe}-\mathrm{C}$ bonds display large transverse relative vibrations and contribute to NTE. However, it can be observed that the main contribution comes from the Ga-N thermal vibrations. Figure taken from Ref. ${ }^{[113]}$. (c) ACS Publishing. Reproduced with permission. All rights reserved.
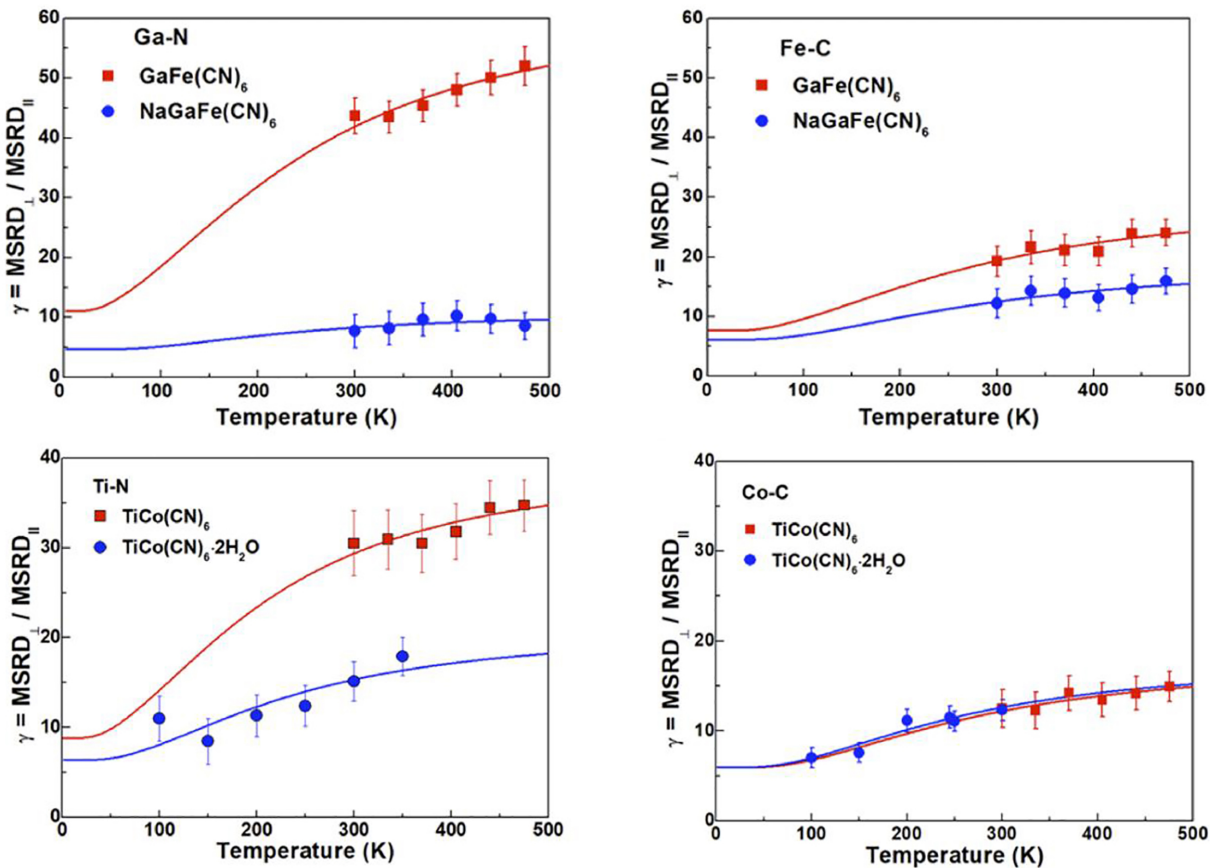

Figure 18. Anisotropy of relative thermal vibrations determined by EXAFS in $\mathrm{Na}: \mathrm{GaFe}(\mathrm{CN})_{6}$ (top panels) and $\mathrm{TiCo}(\mathrm{CN})_{6}: 2 \mathrm{H}_{2} \mathrm{O}($ bottom panels) for the M-N bonds (left panels) and for the $\mathrm{M}^{\prime}-\mathrm{C}$ bonds (right panels). The insertion of guest $\mathrm{Na}^{+}$ions or $\mathrm{H}_{2} \mathrm{O}$ molecules hinders the transverse atomic vibrations, thus inhibiting the NTE. The effect is much more pronounced for the M-N bonds than for the $\mathrm{M}^{\prime}-\mathrm{C}$ bonds.

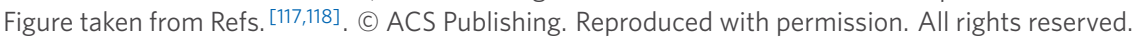



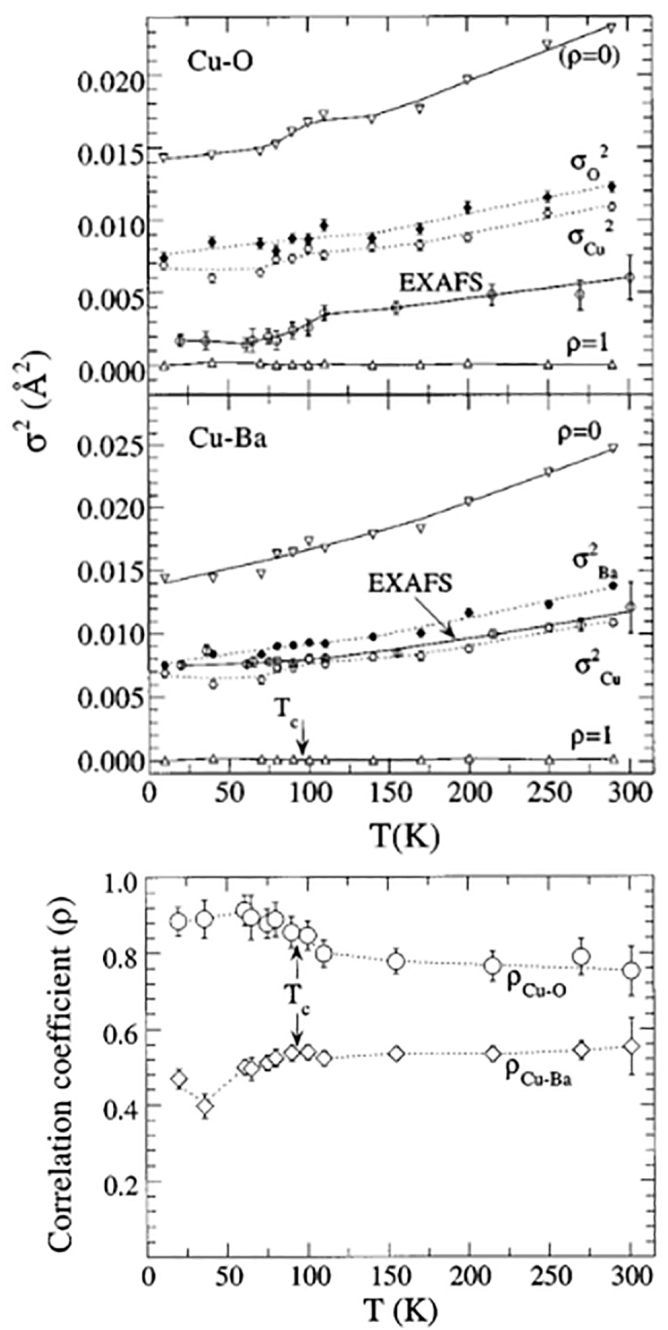

Figure 19. Temperature dependence of the EXAFS Debye-Waller factors (MSRDs) for the $\mathrm{Cu}-\mathrm{O}$ and $\mathrm{Cu}-\mathrm{Ba}$ atomic $\mathrm{pairs}$ in $\mathrm{HgBa}_{2} \mathrm{CuO}_{4+\delta}$ (open circles in top two panels). The diffraction Debye-Waller factors (MSD) $\sigma_{\mathrm{Cu}}{ }^{2}$ for $\mathrm{Cu}$ atoms (open diamonds) and $\sigma_{\mathrm{Ba}}{ }^{2}$ and $\sigma_{\mathrm{O}}{ }^{2}$ for Ba and $\mathrm{O}$ atoms (solid diamonds) are also plotted in the two panels. The Debye-Waller factors calculated from diffraction data for a perfectly correlated $(\rho=1)$ and uncorrelated $(\rho=0)$ motion are shown by up and down triangles, respectively. The lines are guides to the eye. The bottom panel shows the temperature dependence of the correlation coefficient for the $\mathrm{Cu}-\mathrm{O}$ and $\mathrm{Cu}$-Ba distances determined by comparing EXAFS and neutron diffraction Debye-Waller factors [refer to Equation (16)]. Figure taken from Ref. ${ }^{[119] . ~(c) ~ A P S ~ P u b l i s h i n g . ~ R e p r o d u c e d ~}$ with permission. All rights reserved.

agreement with the strong covalent nature of this bond. More importantly, the temperature dependence of the Fe-As MSRDs was found to follow the correlated Einstein model, but with different Einstein frequencies for the superconducting and non-superconducting samples, with a hardening of the Fe-As bond in the former compared to the latter. This finding shows the non-negligible role of the lattice vibrational modes in the superconductivity of pnictide superconductors. This conclusion was supported by Chu et al. ${ }^{[121]}$, who studied the Fe isotopic effect in the $(\mathrm{Ba}, \mathrm{K}) \mathrm{Fe}_{2} \mathrm{As}_{2}$ superconductor with temperature-dependent EXAFS spectroscopy. The authors adopted the Einstein model to separate the static and dynamic isotope contributions. The results show how the isotope substitution has a negligible effect on the interatomic distances but a strong influence on the local vibrations of the Fe-As and Fe-Fe bonds, thus confirming that phonons play an important role in the superconductive mechanism of iron-based superconductor materials. Paris et al. ${ }^{[122]}$ investigated the local atomic displacements in the new $\mathrm{Li}_{\mathrm{x}}\left(\mathrm{NH}_{3}\right)_{\mathrm{y}} \mathrm{Fe}_{2} \mathrm{Se}_{2}$ superconductor and direct information on the Fe-Se and $\mathrm{Fe}-\mathrm{Fe}$ bonds was obtained. The anomalous changes observed in the MSRDs again suggest that the local atomic displacements have a direct relationship with the superconductivity. Other interesting works that show 
the capability of EXAFS in studying the dynamics of superconducting systems can be found in Refs ${ }^{[123-125]}$.

\subsection{Other applications}

EXAFS allows for the exploration of a wide range of topics in condensed-matter physics and materials science. Before concluding, some more examples of EXAFS applications in studying local dynamics are presented.

EXAFS is widely used to study the structural and dynamic aspects of magnetic, ferroelectric and multiferroic materials, as well as of glasses and amorphous systems. Yokoyama et al. ${ }^{[126]}$ studied the spin-crossover transition of a chain Fe(II) complex by temperature-dependent EXAFS and found an abrupt change in the first-shell Fe-N bond distance and the Debye-Waller factor at the transition temperature. The variation of the effective force constant for the Fe- $\mathrm{N}$ stretching vibration revealed a significant weakening of the Fe- $\mathrm{N}$ bond in the high-spin state. Later, the same authors investigated the anharmonicity and quantum effects in the Invar alloy $\mathrm{Fe}_{64.6} \mathrm{Ni}_{35.4}{ }^{[127]}$. The first nearest-neighbor shells around $\mathrm{Fe}$ and $\mathrm{Ni}$ both show a suppression of the bond thermal expansion, although the anharmonicity, with important quantum effects at low temperature, is clearly evident. Panchwanee et al. ${ }^{[128]}$ explored the local modifications associated with the spin-reorientation transition in $\mathrm{DyFeO}_{3}$ orthoferrite. They observed a shrinkage of the $\mathrm{Fe}-\mathrm{O}$ bond length above the spin-reorientation transition and the activation of static distortion of Fe-O bonds below. Smaller effects were observed around Dy atoms.

Fischer et al. ${ }^{[129]}$ detected the antiferrodistortive transition in perovskite $\mathrm{SrTiO}_{3}$ by the temperature-dependence of the EXAFS Debye-Waller factor, which also revealed an anomalous maximum disorder in the lattice vibrations at $31 \mathrm{~K}$. Sanson et al. ${ }^{[130]}$ studied the local vibrational dynamics of hematite $\alpha$ - $\mathrm{Fe}_{2} \mathrm{O}_{3}$. The short and long nearest-neighbor Fe-O distances were singled out and no evidence of the Morin transition was observed. It was found that the short Fe-O bond is stiffer to stretching and softer to bending than the long Fe-O bond. Miyanaga et al. ${ }^{[131]}$ studied the short-range order around $\mathrm{Pb}$ in $\mathrm{PbTiO}_{3}$ from $20 \mathrm{~K}$ to above the Curie temperature. The shorter and medium $\mathrm{Pb}-\mathrm{O}$ nearest-neighbor distances show an order-disorder transition with no significant changes in the bond length across the Curie temperature. In contrast, the longer $\mathrm{Pb}-\mathrm{O}$ distance decreases abruptly and exhibits a displacive transition, with a MSRD that displays a soft mode behavior. As a final example, Subías et al. ${ }^{[132]}$ investigated the first oxygen coordination shell at the manganese site in mixedvalence perovskite manganites as a function of temperature. In the metallic-ferromagnetic phase, manganese is surrounded by regular oxygen octahedra, but above the magnetic transition, the EXAFS Debye-Waller factor shows an increased disorder for the Mn-O distances. Since no structural distortion was detected by the diffraction measurements, this disorder must be dynamic, thus supporting the presence of a large phonon-electronic interaction.

Regarding glass and amorphous systems, in one of the pioneering works, Yang et al. ${ }^{[133]}$ used temperaturedependent EXAFS to provide information about the nature and strength of local bonds of arsenic and arsenicchalcogen compounds. They found that the As-As bonds in c-As are weaker than those in a-As, while the stretching forces of the As-S bonds in crystalline and glass $\mathrm{As}_{2} \mathrm{~S}_{3}$ are very similar. In contrast, Sanson et al. ${ }^{[134]}$ found a softening of the Mo-O bonds in $\mathrm{AgI}: \mathrm{Ag}_{2} \mathrm{MoO}_{4}$ glasses with respect to the crystalline counterpart $\mathrm{Ag}_{2} \mathrm{MoO}_{4}$. In the same glasses, EXAFS cumulants were employed to monitor the progressive depletion of the short-range I-Ag distance distribution, due to the transitions of Ag ions into a long-range component, thus obtaining information on the local modifications around iodine due to the ionic diffusion ${ }^{[135]}$. Siqueira et al. ${ }^{[136]}$ investigated the structural and thermal properties in an amorphous $\mathrm{GaSe}_{9}$ alloy by combining EXAFS and reverse Monte Carlo simulations, thus obtaining information on the interatomic distances, thermal disorder, asymmetry of the distance distribution and anharmonicity of the effective interatomic pair potential. Yoshitake et al. ${ }^{[137]}$ studied amorphous mesoporous titania with EXAFS to clarify the characteristics of the Ti-O chemical bonds. The coexistence of 5- and 6-fold coordinated Ti was demonstrated and the EXAFS Debye-Waller factor suggested that the pores filled with template micelles suppress the thermal vibrations of 
Ti-O bonds.

The examples given in this section are clearly not exhaustive and other EXAFS studies of local dynamics can be found in the literature.

\section{CONCLUSION}

This paper has presented a brief review on the potential of EXAFS for the study of the local vibrational dynamics in materials. The strength of EXAFS is the selectivity of the atomic species and the sensitivity to short-range order and correlation of atomic motion.

The EXAFS approach, based on the cumulant expansion, gives information on the distribution of distances between the atoms of the absorbing species and their nearest neighbors, including the deviations from a Gaussian shape. In addition to giving information on the distance distribution and the anharmonicity of the interatomic potential, the EXAFS cumulants provide information on the local vibrational dynamics. Specifically, the first cumulant, benefitting from the difference between EXAFS and diffraction, leads to the evaluation of the perpendicular MSRD, while the second cumulant corresponds, in good approximation, to the parallel MSRD. The anisotropy of the relative thermal vibrations can thus be determined by EXAFS.

Some information has been provided regarding the data analysis strategy in order to improve the EXAFS results. In particular, the binding energy $E_{0}$ and the loss factor $S_{0}^{2}$ play a key role in determining the trend of the EXAFS cumulants. The values of $E_{0}$ and $S_{0}{ }^{2}$ heavily influence the absolute values of the odd and even cumulants, respectively. If possible, the third and fourth cumulants should be included in the EXAFS analysis to obtain much more precise results for the first and second cumulants, respectively.

Finally, in order to show the capability of EXAFS to study the local dynamics, several examples of application have been reported, ranging over a wide array of topics. This work encourages the use of EXAFS spectroscopy as a dynamic probe and not only as a structural probe.

\section{DECLARATIONS}

\section{Authors' contributions}

The author contributed solely to the article.

\section{Availability of data and materials}

Not applicable.

\section{Financial support and sponsorship}

None.

\section{Conflicts of interest}

The author declared that there are no conflicts of interest.

\section{Ethical approval and consent to participate}

Not applicable.

\section{Consent for publication}

Not applicable. 


\section{Copyright}

(c)The Author (s) 2021.

\section{REFERENCES}

1. O'connor DA. Thermal vibrations in crystallography. Phys Bull 1975;26:498-9.

2. Artioli G. Atomic displacement parameters from diffraction studies. In: Gramaccioli CM, Papp G, Weiszburg T, editors. Energy modelling in minerals. budapest: mineralogical society of Great Britain and Ireland; 2002. p. 389-405.

3. Wood IG, Knight KS, Price GD, Stuart JA. Thermal expansion and atomic displacement parameters of cubic $\mathrm{KMgF}_{3}$ perovskite determined by high-resolution neutron powder diffraction. J Appl Crystallogr 2002;35:291-5.

4. Denisov VN, Ivlev AN, Lipin AS, Mavrin BN, Orlov VG. Raman spectra and lattice dynamics of single-crystal. J Phys: Condens Matter 1997;9:4967-78.

5. Maczka M, Hanuza J, Paraguassu W, Gomes Souza Filho A, Tarso Cavalcante Freire P, Mendes Filho J. Phonons in ferroelectric Bi2WO6: Raman and infrared spectra and lattice dynamics. Appl Phys Lett 2008;92:112911.

6. Birman JL. Theory of crystal space groups and infra-red and raman lattice processes of insulating crystals. Theory of crystal space groups and lattice dynamics. Berlin: Springer Berlin Heidelberg; 1984. p. 1-521.

7. Strauch D, Pavone P, Mayer AP, et al. Ab initio lattice dynamics: methods, results, and applications. In: Helbig R, editor. Advances in solid state physics 37. Berlin: Springer Berlin Heidelberg; 1998. p. 99-124.

8. Gonze X, Rignanese G, Caracas R. First-principle studies of the lattice dynamics of crystals, and related properties. Zeitschrift für Kristallographie - Crystalline Materials 2005;220:458-72.

9. Dove MT. Some fundamentals. Introduction to Lattice Dynamics. Cambridge University Press; 2010. p. 1-17.

10. Albertsson J, Abrahams SC, Kvick $\AA$. Atomic displacement, anharmonic thermal vibration, expansivity and pyroelectric coefficient thermal dependences in ZnO. Acta Crystallogr B Struct Sci 1989;45:34-40.

11. Downs RT, Gibbs GV, Boisen MB. A study of the mean-square displacement amplitudes of $\mathrm{Si}, \mathrm{Al}$, and $\mathrm{O}$ atoms in framework structures; evidence for rigid bonds, order, twinning, and stacking faults. American Mineralogist 1990;75:1253-67.

12. Krisch M, Sette F. Inelastic X-Ray Scattering from Phonons. In: Cardona M, Merlin R, editors. Light scattering in solid IX. Berlin: Springer Berlin Heidelberg; 2006. p. 317-70.

13. Burkel E. Phonon spectroscopy by inelastic x-ray scattering. Rep Prog Phys 2000;63: 171-232.

14. 14. Dickens MH, Hutchings MT. Inelastic neutron scattering study of the phonon dispersion relation of $\mathrm{PbF}_{2}$ at $10 \mathrm{~K}$. J Phys C: Solid State Phys 1978;11:461-8.

15. Rüffer R, Chumakov AI. Nuclear inelastic scattering. Hyperfine Interactions 2000;128:255-72.

16. Giarola M, Sanson A, Monti F, et al. Vibrational dynamics of anatase TiO2: Polarized Raman spectroscopy and ab initio calculations. Phys Rev B 2010;81.

17. Iliev MN, Lee H, Popov VN, et al. Raman- and infrared-active phonons in hexagonal YMnO3: Experiment and lattice-dynamical calculations. Phys Rev B 1997;56:2488-94.

18. Stern EA, Sayers DE, Lytle FW. Extended x-ray-absorption fine-structure technique. III. Determination of physical parameters. Phys Rev B 1975;11:4836-46.

19. Beni G, Platzman PM. Temperature and polarization dependence of extended x-ray absorption fine-structure spectra. Phys Rev $B$ 1976;14:1514-8.

20. Fornasini P. Study of lattice dynamics via extended x-ray absorption fine structure. J Phys: Condens Matter 2001;13:7859-72.

21. Schnohr CS, Kluth P, Araujo LL, et al. Anisotropic vibrations in crystalline and amorphous InP. Phys Rev B 2009;79.

22. Dalba G, Fornasini P. EXAFS Debye-Waller factor and thermal vibrations of crystals. J Synchrotron Radiat 1997;4:243-55.

23. Teo B. Extended X-Ray Absorption Fine Structure (EXAFS) spectroscopy: techniques and applications. In: Teo BK, Joy DC, editors. EXAFS spectroscopy. Boston: Springer US; 1981. p. 13-58.

24. Paufler PP. Introduction to XAFS. A practical guide to X-ray absorption fine structure spectroscopy. By Grant Bunker. p. viii +260 . Cambridge University Press, 2010. Price (hardback) GBP 40. ISBN-13: 978-0-521-76775-0. J Synchrotron Rad 2011;18:818.

25. Crozier RED, Rehr JJ, Ingalls R. X-ray absorption: principles, applications, techniques of exafs, sexafs, and xanes. New York: Wiley, 1988.

26. Hayes T, Boyce J. Extended X-ray absorption fine structure spectroscopy. Elsevier; 1983. p. 173-351.

27. Henderson GS, de Groot FMF, Moulton BJA. X-ray absorption near-edge structure (XANES) spectroscopy. Reviews in Mineralogy and Geochemistry 2014;78:75-138.

28. Newville M. Fundamentals of XAFS. Reviews in Mineralogy and Geochemistry 2014;78: 33-74.

29. Bunker G. Application of the ratio method of EXAFS analysis to disordered systems. Nucl Instrum Methods Phys Res A 1983;207:43744.

30. Fornasini P, Monti F, Sanson A. On the cumulant analysis of EXAFS in crystalline solids. J Synchrotron Radiat 2001;8:1214-20.

31. Busing WR, Levy HA. The effect of thermal motion on the estimation of bond lengths from diffraction measurements. Acta Cryst $1964 ; 17: 142-6$.

32. Vaccari M, Grisenti R, Fornasini P, Rocca F, Sanson A. Negative thermal expansion in $\mathrm{CuCl}$ : an extended x-ray absorption fine structure study. Phys Rev B 2007;75. 
33. Ahmed SI, Aquilanti G, Novello N, Olivi L, Grisenti R, Fornasini P. Local vibrational properties of GaAs studied by extended X-ray absorption fine structure. J Chem Phys 2013;139:164512.

34. Yoshiasa A, Koto K, Maeda H, Ishii T. The mean-square relative displacement and displacement correlation functions in tetrahedrally and octahedrally coordinated A(N)B(8-N) crystals. Jpn J Appl Phys 1997;36:781-4.

35. Sanson A, Rocca F, Dalba G, et al. Negative thermal expansion and local dynamics in Cu2O and Ag2O. Phys Rev B 2006;73.

36. Fornasini P, a Beccara S, Dalba G, et al. Extended x-ray-absorption fine-structure measurements of copper: local dynamics, anharmonicity, and thermal expansion. Phys Rev B 2004;70.

37. Dalba G, Fornasini P, Grisenti R, Purans J. Sensitivity of extended x-ray-absorption fine structure to thermal expansion. Phys Rev Lett 1999;82:4240-3.

38. Sanson A. Local dynamical properties of crystalline germanium and their effects in extended x-ray absorption fine structure. Phys Rev B 2010; 81 .

39. Cusack NE, Stein DL. The physics of structurally disordered matter: an introduction. Physics Today 1988;41:110-2.

40. Tranquada JM, Ingalls R. Extended x-ray - absorption fine-structure study of anharmonicity in CuBr. Phys Rev B 1983;28:3520-8.

41. Stern EA, Livns P, Zhang Z. Thermal vibration and melting from a local perspective. Phys Rev B Condens Matter 1991;43:8850-60.

42. Dalba G, Fornasini P, Grisenti R, Pasqualini D, Diop D, Monti F. Anharmonicity effects on the extended X-ray-absorption fine structure: the case of cadmium selenide. Phys Rev B 1998;58:4793-802.

43. Sanson A. Bond thermal expansion and effective pair potential in crystals: the case of cadmium selenide. J Phys Condens Matter 2011;23:315401.

44. Comaschi T, Balerna A, Mobilio S. Thermal dependent anharmonicity effects on gold bulk studied by extended x-ray-absorption fine structure. J Phys Condens Matter 2009;21:325404.

45. Kamishima O, Ishii T, Maeda H, Kashino S. EXAFS study on temperature dependence of nearest neighbor distance in CuBr. Solid State Communications 1997;103:141-4.

46. Dalba G, Fornasini P, Gotter R, Rocca F. Anharmonicity effects on the extended x-ray-absorption fine structure: the case of beta-AgI Phys Rev B Condens Matter 1995;52:149-57.

47. Frenkel AI, Rehr JJ. Thermal expansion and x-ray-absorption fine-structure cumulants. Phys Rev B Condens Matter 1993;48:585-8.

48. Yokoyama T. Path-integral effective-potential theory for EXAFS cumulants compared with the second-order perturbation. J Synchrotron Radiat 1999;6:323-5.

49. Van Hung N, Rehr JJ. Anharmonic correlated Einstein-model Debye-Waller factors. Phys Rev B 1997;56:43-6.

50. Van Hung N, Fornasini P. Anharmonic effective potential, correlation effects, and EXAFS cumulants calculated from a morse interaction potential for fcc metals. J Phys Soc Jpn 2007;76:084601.

51. Hung NV, Tien TS, Duc NB, Vuong DQ. High-order expanded XAFS Debye-Waller factors of HCP crystals based on classical anharmonic correlated Einstein model. Mod Phys Lett B 2014;28:1450174.

52. Vila FD, Lindahl VE, Rehr JJ. X-ray absorption Debye-Waller factors from ab initio molecular dynamics. Phys Rev B 2012;85.

53. Miyanaga T, Fujikawa T. Quantum statistical approach to Debye-Waller factors in EXAFS, EELS and ARXPS. VI. Path-integral approach to morse potential systems. J Phys Soc Jpn 1998;67:2930-7.

54. Rehr JJ, Albers RC. Theoretical approaches to x-ray absorption fine structure. Rev Mod Phys 2000;72:621-54.

55. Rehr JJ, Kas JJ, Vila FD, Prange MP, Jorissen K. Parameter-free calculations of X-ray spectra with FEFF9. Phys Chem Chem Phys 2010;12:5503-13.

56. Purans J, Afify ND, Dalba G, et al. Isotopic effect in extended x-ray-absorption fine structure of germanium. Phys Rev Lett 2008;100:055901.

57. Sanson A. Isotopic effect on the local dynamics of crystalline germanium. Solid State Sciences 2010;12:1988-92.

58. Yokoyama T, Ohta T, Sato H. Thermal expansion and anharmonicity of solid Kr studied by extended x-ray-absorption fine structure. Phys Rev B 1997;55:11320-9.

59. Sanson A. On the neglecting of higher-order cumulants in EXAFS data analysis. J Synchrotron Radiat 2009;16:864-8.

60. Jeong I, Heffner RH, Graf MJ, Billinge SJL. Lattice dynamics and correlated atomic motion from the atomic pair distribution function. Phys Rev B 2003;67.

61. Beccara SA, Fornasini P. Path-integral Monte Carlo calculation of the effects of thermal disorder in extended x-ray-absorption fine structure of copper. Phys Rev B 2008;77.

62. Maradudin AA, Montroll EW, Weiss GH, Ipatova, IP, Theory of lattice dynamics in the harmonic approximation. New York: Academic Press, 1971.

63. Sevillano E, Meuth H, Rehr JJ. Extended x-ray absorption fine structure Debye-Waller factors. I. Monatomic crystals. Phys Rev B 1979;20:4908-11.

64. Vaccari M, Fornasini P. Einstein and Debye models for EXAFS parallel and perpendicular mean-square relative displacements. $J$ Synchrotron Radiat 2006;13:321-5.

65. Hu L, Chen J, Sanson A, et al. New insights into the negative thermal expansion: direct experimental evidence for the "Guitar-String" effect in cubic ScF3. J Am Chem Soc 2016;138:8320-3.

66. Li CW, Tang X, Muñoz JA, et al. Structural relationship between negative thermal expansion and quartic anharmonicity of cubic ScF3. Phys Rev Lett 2011;107:195504.

67. Sanson A. On the Einstein model for EXAFS parallel and perpendicular mean-square relative displacements. $J$ Synchrotron Radiat 2008;15:514-8. 
68. Timoshenko J, Anspoks A, Kalinko A, Kuzmin A. Local structure and dynamics of wurtzite-type ZnO from simulation-based EXAFS analysis: local structure and dynamics of wurtzite-type ZnO from simulation-based EXAFS analysis. Phys Status Solidi C 2014;11:14725.

69. Jonane I, Lazdins K, Timoshenko J, et al. Temperature-dependent EXAFS study of the local structure and lattice dynamics in cubic $\mathrm{Y}_{2} \mathrm{O}_{3}$. J Synchrotron Radiat 2016;23:510-8.

70. Timoshenko J, Kuzmin A, Purans J. Molecular dynamics simulations of EXAFS in germanium. Open Physics 2011; 9.

71. Yokoyama T. Path-integral effective-potential method applied to extended x-ray-absorption fine-structure cumulants. Phys Rev $B$ 1998;57:3423-32.

72. Beccara S, Dalba G, Fornasini P, et al. Local thermal expansion in copper: Extended x-ray-absorption fine-structure measurements and path-integral Monte Carlo calculations. Phys Rev B 2003;68.

73. Palmer BJ, Pfund DM, Fulton JL. Direct modeling of EXAFS spectra from molecular dynamics simulations. J Phys Chem 1996;100:13393-8.

74. Price SWT, Zonias N, Skylaris C, Hyde TI, Ravel B, Russell AE. Fitting EXAFS data using molecular dynamics outputs and a histogram approach. Phys Rev B 2012;85.

75. Kuzmin A, Efimov V, Efimova E, Sikolenko V, Pascarelli S, Troyanchuk I. Interpretation of the Co K-edge EXAFS in LaCoO3 using molecular dynamics simulations. Solid State Ionics 2011;188:21-4.

76. Bocharov D, Krack M, Rafalskij Y, Kuzmin A, Purans J. Ab initio molecular dynamics simulations of negative thermal expansion in ScF3: the effect of the supercell size. Computational Materials Science 2020;171:109198.

77. Sanson A. Toward an understanding of the local origin of negative thermal expansion in $\mathrm{ZrW}_{2} \mathrm{O}_{8}$ : limits and inconsistencies of the tent and rigid unit mode models. Chem Mater 2014;26:3716-20.

78. Bocharov D, Anspoks A, Timoshenko J, Kalinko A, Krack M, Kuzmin A. Interpretation of the Cu K-edge EXAFS spectra of Cu3N using ab initio molecular dynamics. Radiation Physics and Chemistry 2020;175:108100.

79. Roscioni OM, Zonias N, Price SWT, Russell AE, Comaschi T, Skylaris C. Computational prediction of L3 EXAFS spectra of gold nanoparticles from classical molecular dynamics simulations. Phys Rev B 2011;83.

80. Mierzwa B. EXAFS studies of bimetallic palladium-cobalt nanoclusters using Molecular Dynamics simulations. $J$ Alloys Compd 2005;401:127-34.

81. Anspoks A, Kuzmin A. Interpretation of the Ni K-edge EXAFS in nanocrystalline nickel oxide using molecular dynamics simulations. J Non Cryst Solids 2011;357:2604-10.

82. Rybicki J, Rybicka A, Witkowska A, et al. The structure of lead-silicate glasses: molecular dynamics and EXAFS studies. $J$ Phys: Condens Matter 2001;13:9781-97.

83. Rossano S, Ramos A, Delaye JM, et al. Iron surrounding in $\mathrm{CaO}-\mathrm{FeO}-2 \mathrm{SiO} 2$ glass: EXAFS and molecular dynamics simulation. $J$ Synchrotron Radiat 1999;6:247-8.

84. Dziegielewski P, Mathon O, Kantor I, et al. High pressure atomic structure of Zr-Cu metallic glass via EXAFS spectroscopy and molecular dynamics simulations. High Pressure Research 2020;40:54-64.

85. Migliorati V, Serva A, Aquilanti G, et al. Combining EXAFS spectroscopy and molecular dynamics simulations to understand the structural and dynamic properties of an imidazolium iodide ionic liquid. Phys Chem Chem Phys 2015;17:2464-74.

86. Serva A, Migliorati V, Spezia R, D'Angelo P. How does Ce $\mathrm{III}^{\mathrm{III}}$ nitrate dissolve in a protic ionic liquid? a combined molecular dynamics and EXAFS study. Chemistry 2017;23: 8424-33.

87. Busato M, Lapi A, D'Angelo P, Melchior A. Coordination of the $\mathrm{Co}^{2+}$ and $\mathrm{Ni}^{2+}$ ions in $\mathrm{Tf}_{2} \mathrm{~N}^{-}$based ionic liquids: a combined $\mathrm{x}$-ray absorption and molecular dynamics study. J Phys Chem B 2021;125:6639-48.

88. Rockenberger J, Tröger L, Kornowski A, et al. EXAFS studies on the size dependence of structural and dynamic properties of CdS nanoparticles. J Phys Chem B 1997;101:2691-701.

89. Araujo LL, Kluth P, de M. Azevedo G, Ridgway MC. Vibrational properties of Ge nanocrystals determined by EXAFS. Phys Rev B $2006 ; 74$.

90. Gilbert B, Huang F, Zhang H, Waychunas GA, Banfield JF. Nanoparticles: strained and stiff. Science 2004;305:651-4.

91. Rockenberger J, Tröger L, Rogach AL, et al. The contribution of particle core and surface to strain, disorder and vibrations in thiolcapped CdTe nanocrystals. J Chem Phys 1998;108:7807-15.

92. Sprouster DJ, Giulian R, Araujo LL, et al. Structural and vibrational properties of Co nanoparticles formed by ion implantation. $J$ Appl Phys 2010;107:014313.

93. Comaschi T, Balerna A, Mobilio S. Temperature dependence of the structural parameters of gold nanoparticles investigated with EXAFS. Phys Rev B 2008;77.

94. Li WH, Wu SY, Yang CC, et al. Thermal contraction of au nanoparticles. Phys Rev Lett 2002;89:135504.

95. Duan Z, Li Y, Timoshenko J, et al. A combined theoretical and experimental EXAFS study of the structure and dynamics of $\mathrm{Au}_{147}$ nanoparticles. Catal Sci Technol 2016;6: 6879-85.

96. Timoshenko J, Duan Z, Henkelman G, Crooks RM, Frenkel AI. Solving the structure and dynamics of metal nanoparticles by combining X-Ray absorption fine structure spectroscopy and atomistic structure simulations. Annu Rev Anal Chem (Palo Alto Calif) 2019;12:50122.

97. Hu L, Qin F, Sanson A, et al. Localized symmetry breaking for tuning thermal expansion in $\mathrm{ScF}_{3}$ nanoscale frameworks. $J$ Am Chem Soc 2018;140:4477-80.

98. Mary TA, Evans JSO, Vogt T, Sleight AW. Negative thermal expansion from 0.3 to 1050 Kelvin in ZrW2O8. Science 1996;272:90-2. 
99. Cai W, Katrusiak A. Giant negative linear compression positively coupled to massive thermal expansion in a metal-organic framework. Nat Commun 2014;5:4337.

100. Chen J, Hu L, Deng J, Xing X. Negative thermal expansion in functional materials: controllable thermal expansion by chemical modifications. Chem Soc Rev 2015;44:3522-67.

101. Cao D, Bridges F, Kowach GR, Ramirez AP. Frustrated soft modes and negative thermal expansion in ZrW2O8. Phys Rev Lett 2002;89:215902.

102. Cao D, Bridges F, Kowach GR, Ramirez AP. Correlated atomic motions in the negative thermal expansion material ZrW2O8: a local structure study. Phys Rev B 2003;68.

103. a Beccara S, Dalba G, Fornasini P, Grisenti R, Sanson A, Rocca F. Local thermal expansion in a cuprite structure: the case of $\mathrm{Ag}(2) \mathrm{O}$. Phys Rev Lett 2002;89:025503.

104. Dapiaggi M, Tiano W, Artioli G, Sanson A, Fornasini P. The thermal behaviour of cuprite: An XRD-EXAFS combined approach. Nucl Instrum Methods Phys Res B 2003;200:231-6.

105. Hammonds KD, Dove MT, Giddy AP, Heine V, Winkler B. Rigid-unit phonon modes and structural phase transitions in framework silicates. American Mineralogist 1996;81:1057-79.

106. Sanson A. Tension effect in local dynamics of cuprite structures. Solid State Sciences 2009;11:1489-93.

107. Abd el All N, Dalba G, Diop D, et al. Negative thermal expansion in crystals with the zincblende structure: an EXAFS study of CdTe. $J$ Phys Condens Matter 2012;24:115403.

108. Barrera GD, Bruno JAO, Barron THK, Allan NL. Negative thermal expansion. J Phys: Condens Matter 2005;17:R217-52.

109. Qin F, Chen J, Aydemir U, et al. Isotropic zero thermal expansion and local vibrational dynamics in ( $\mathrm{Sc}, \mathrm{Fe}) \mathrm{F}_{3}$. Inorg Chem 2017;56:10840-3.

110. Purans J, Fornasini P, Ali SE, Dalba G, Kuzmin A, Rocca F. X-ray absorption spectroscopy study of local dynamics and thermal expansion in ReO3. Phys Rev B 2015;92.

111. Zakaria MB, Chikyow T. Recent advances in Prussian blue and Prussian blue analogues: synthesis and thermal treatments. Coordination Chemistry Reviews 2017;352:328-45.

112. Chapman KW, Chupas PJ, Kepert CJ. Compositional dependence of negative thermal expansion in the prussian blue analogues MIIPtIV(CN)6 (M: Mn, Fe, Co, Ni, Cu, Zn, Cd). ChemInform 2006;37.

113. Gao Q, Shi N, Sun Q, et al. Low-frequency phonon driven negative thermal expansion in cubic $\mathrm{GaFe}(\mathrm{CN})_{6}$ prussian blue analogues. Inorg Chem 2018;57:10918-24.

114. Shi N, Gao Q, Sanson A, et al. Negative thermal expansion in cubic FeFe(CN) 6 Prussian blue analogues. Dalton Trans 2019;48:3658-63.

115. Gao Q, Sun Y, Shi N, et al. Large isotropic negative thermal expansion in water-free Prussian blue analogues of $\mathrm{ScCo}(\mathrm{CN}) 6$. Scripta Materialia 2020;187:119-24.

116. Gao Q, Chen J, Sun Q, et al. Switching between giant positive and negative thermal expansions of a $\mathrm{YFe}(\mathrm{CN})_{6}$-based prussian blue analogue induced by guest species. Angew Chem Int Ed Engl 2017;56:9023-8.

117. Gao Q, Shi N, Sanson A, et al. Tunable thermal expansion from negative, zero, to positive in cubic prussian blue analogues of GaFe $(\mathrm{CN})_{6}$. Inorg Chem 2018;57:14027-30.

118. Gao Q, Shi X, Venier A, et al. Effect of $\mathrm{H}_{2} \mathrm{O}$ molecules on thermal expansion of TiCo(CN) 6 . Inorg Chem 2020;59:14852-5.

119. Lanzara A, Saini NL, Bianconi A, Duc F, Bordet P. Anomalous local atomic correlations in HgBa2CuO4+ . Phys Rev B 1999;59:3851-4

120. Joseph B, Iadecola A, Malavasi L, Saini NL. Temperature-dependent local structure of $\mathrm{NdFeAsO}(1-\mathrm{x}) \mathrm{F}(\mathrm{x})$ system using arsenic K-edge extended x-ray absorption fine structure. J Phys Condens Matter 2011;23:265701.

121. Chu W, Cheng J, Chu S, et al. Iron isotope effect and local lattice dynamics in the (Ba, K)Fe2As2 Superconductor studied by temperaturedependent EXAFS. Sci Rep 2013; 3.

122. Paris E, Simonelli L, Wakita T, et al. Temperature dependent local atomic displacements in ammonia intercalated iron selenide superconductor. Sci Rep 2016;6:27646.

123. Saini NL, Oyanagi H, Ito T, et al. Temperature dependent local Cu-O displacements from underdoped to overdoped La-Sr-Cu-O superconductor. The European Physical Journal B - Condensed Matter 2003;36:75-80.

124. Saini NL, Filippi M, Oyanagi H, Ihara H, Iyo A, Bianconi A. Temperature-dependent local structure in the Nb3Ge superconductor studied by high-resolution Ge K -edge EXAFS measurements. Phys Rev B 2003;68.

125. Chu WS, Zhang S, Yu MJ, et al. Correlation between local vibrations and metal mass in AlB2-type transition-metal diborides. $J$ Synchrotron Radiat 2009;16:30-7.

126. Yokoyama T, Murakami Y, Kiguchi M, Komatsu T, Kojima N. Spin-crossover phase transition of a chain Fe(II) complex studied by X-ray-absorption fine-structure spectroscopy. Phys Rev B 1998;58:14238-44.

127. Yokoyama T, Eguchi K. Anharmonicity and quantum effects in thermal expansion of an Invar alloy. Phys Rev Lett 2011;107:065901.

128. Panchwanee A, Schiesaro I, Mobilio S, et al. An evidence of local structural disorder across spin-reorientation transition in $\mathrm{DyFeO}_{3}$ : an extended x-ray absorption fine structure (EXAFS) study. J Phys Condens Matter 2019;31:345403.

129. Fischer M, Lahmar A, Maglione M, et al. Local disorder studied in SrTiO3 at low temperature by EXAFS spectroscopy. Phys Rev B Condens Matter 1994;49:12451-6.

130. Sanson A, Mathon O, Pascarelli S. Local vibrational dynamics of hematite $\left(\alpha-\mathrm{Fe}_{2} \mathrm{O}_{3}\right)$ studied by extended $\mathrm{x}$-ray absorption fine structure and molecular dynamics. $J$ Chem Phys 2014;140:224504.

131. Miyanaga T, Diop D, Ikeda S, Kon H. Study of the local structure changes in PbTiO 3 by Pb L III EXAFS. Ferroelectrics 2002;274:41-53.

132. Subías G, García J, Blasco J, Proietti MG. Local structure at the manganese site in mixed-valence manganites. Phys Rev B 1998;57:748- 
54.

133. Yang CY, Paesler MA, Sayers DE. Determination of bond strengths of arsenic and arsenic chalcogen compounds using the temperature dependence of extended x-ray-absorption fine structure. Phys Rev B Condens Matter 1987;36:980-8.

134. Sanson A, Rocca F, Armellini C, Ahmed S, Grisenti R. Local study on the MoO4 units in AgI-doped silver molybdate glasses. J Non Cryst Solids 2008;354:94-7.

135. Sanson A, Rocca F, Dalba G, Fornasini P, Grisenti R. Influence of temperature on the local structure around iodine in fast-ion-conducting AgI: $\mathrm{Ag}_{2} \mathrm{MoO}_{4}$ glasses. New J Phys 2007;9:88

136. Siqueira MC, Maia RN, Araujo RM, Machado KD, Stolf SF. Structural and thermal investigations of an amorphous GaSe 9 alloy using EXAFS, cumulant expansion, and reverse Monte Carlo simulations. J Chem Phys 2015;142:054504.

137. Yoshitake H, Sugihara T, Tatsumi T .XAFS study on the local structure of Ti in amorphous mesoporous titania. Phys Chem Chem Phys 2003;3:767-72.

138. Strauch D, Pavone P, Nerb N, et al. Atomic thermal vibrations in semiconductors: Ab initio calculations and EXAFS measurements. Physica B: Condensed Matter 1996;219-220:436-8. 